Supporting Information

\title{
Bromoporphyrins as Versatile Synthons for Modular Construction of Chiral Porphyrins: Cobalt-Catalyzed Highly Enantioselective and Diastereoselective Cyclopropanation
}

\author{
Ying Chen, Kimberly B. Fields and X. Peter Zhang* \\ Department of Chemistry, University of Tennessee, Knoxville, TN 37996
}

\begin{abstract}
Experimental Section
General Considerations. All cross-coupling reactions were carried out under a nitrogen atmosphere in oven-dried glassware following standard Schlenk techniques. Tetrahydrofuran (THF) and toluene were distilled under nitrogen from sodium benzophenone ketyl. Chiral amides were purchased from Aldrich Chemical Co. and Acros Organics, used without further purification. Anhydrous cobalt(II) chloride, cobalt acetate tetrahydrate, palladium(II) acetate, and 9,9-dimethyl-4,5-bis(diphenylphosphino)xanthene (Xantphos) were purchased from Strem Chemical Co.. Cesium carbonate was obtained as a gift from Chemetall Chemical Products, Inc..
\end{abstract}

General Procedures for Synthesis of Brominated Porphyrins The brominated porphyrins were prepared according to the method described in literature. ${ }^{1,2}$ A mixture of meso- $(2,6-$ dibromophenyl)dipyrromethane $(1 \mathrm{mmol})$, aldehyde $(1 \mathrm{mmol})$, and molecular sieves (4A, $0.300 \mathrm{~g})$ in chloroform $(150 \mathrm{ml})$ was purged with nitrogen for $10 \mathrm{~min}$. Boron trifluoride diethyl etherate $(0.1 \mathrm{~mL})$ was added dropwise via a syringe and the flask was wrapped with aluminum foil to shield it from light. The solution was stirred under a nitrogen atmosphere at room temperature for $3 \mathrm{~h}$, and 2,3-dichloro-5,6-dicyano-1,4-benzoquinone (DDQ) (1.2 mmol) was added as powder at one time. After $30 \mathrm{~min}, 1 \mathrm{~mL}$ of triethylamine was added. The reaction solution was then directly poured on the top of a silica gel column that was packed with dichloromethane. The column was eluted with dichloromethane. The fractions containing product were collected and concentrated on a rotary evaporator. The residue was washed several times with hexanes to afford the pure compound.

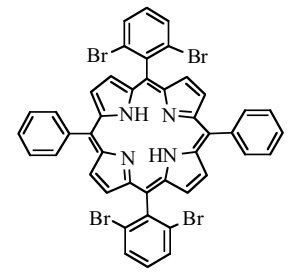

5,15-Bis(2,6-dibromophenyl)-10,20-diphenylporphyrin 1a. Purple solid. Yield: $41 \% .{ }^{1} \mathrm{H}$ NMR (300 MHz, CDCl $): \delta 8.84(\mathrm{~d}, J=4.8 \mathrm{~Hz}, 4 \mathrm{H}), 8.63(\mathrm{~d}, J=4.8 \mathrm{~Hz}, 4 \mathrm{H}), 8.22(\mathrm{~m}, 4 \mathrm{H})$, $8.01(\mathrm{~d}, J=8.1 \mathrm{~Hz}, 4 \mathrm{H}), 7.73(\mathrm{~m}, 6 \mathrm{H}), 7.52(\mathrm{t}, J=8.1 \mathrm{~Hz}, 2 \mathrm{H}),-2.61(\mathrm{~s}, 2 \mathrm{H})$. UV-vis $\left(\mathrm{CH}_{2} \mathrm{Cl}_{2}\right), \lambda_{\max } \mathrm{nm}(\log \varepsilon): 419(5.65), 515(4.31), 548(3.76), 590(3.81), 646(3.41)$. HRMSMALDI $\left([\mathrm{M}+\mathrm{H}]^{+}\right)$: calcd for $\mathrm{C}_{44} \mathrm{H}_{27} \mathrm{Br}_{4} \mathrm{~N}_{4}$ 926.8964; found: 926.8992 with an isotope distribution pattern that is the same as the calculated one. 


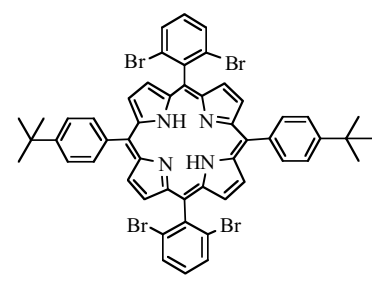

5,15-Bis(2,6-dibromophenyl)-10,20-bis[4-(tert-butyl)phenyl]porphyrin $1 \mathrm{~b}$. Purple solid. Yield: $61 \%$. ${ }^{1} \mathrm{H}$ NMR $\left(300 \mathrm{MHz}, \mathrm{CDCl}_{3}\right): \delta 8.88(\mathrm{~d}, J=4.8 \mathrm{~Hz}, 4 \mathrm{H}), 8.61(\mathrm{~d}, J=4.8 \mathrm{~Hz}$, $4 \mathrm{H}), 8.14(\mathrm{~d}, J=8.1 \mathrm{~Hz}, 4 \mathrm{H}), 8.01(\mathrm{~d}, J=8.1 \mathrm{~Hz}, 4 \mathrm{H}), 7.73(\mathrm{~d}, J=8.1 \mathrm{~Hz}, 4 \mathrm{H}), 7.52(\mathrm{t}, J=$ $8.1 \mathrm{~Hz}, 2 \mathrm{H}), 1.52(\mathrm{~s}, 18 \mathrm{H}),-2.57(\mathrm{~s}, 2 \mathrm{H}) . \mathrm{UV}-\mathrm{vis}\left(\mathrm{CH}_{2} \mathrm{Cl}_{2}\right), \lambda_{\max } \mathrm{nm}(\log \varepsilon): 421(5.68)$, 516(4.31), 551(3.82), 592(3.81), 647(3.47). HRMS-MALDI $\left([\mathrm{M}+\mathrm{H}]^{+}\right)$: calcd for $\mathrm{C}_{52} \mathrm{H}_{43} \mathrm{Br}_{4} \mathrm{~N}_{4}$ 1039.0216; found: 1039.0236 with an isotope distribution pattern that is the same as the calculated one.

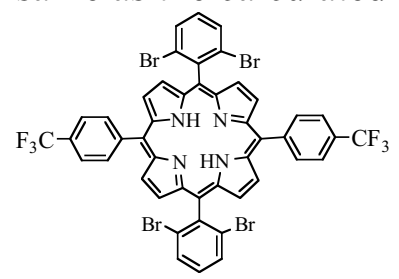

5,15-Bis(2,6-dibromophenyl)-10,20-bis(4-trifluoromethylphenyl)porphyrin 1c. Purple solid. Yield: 45\%. ${ }^{1} \mathrm{H}$ NMR $\left(300 \mathrm{MHz}, \mathrm{CDCl}_{3}\right): \delta 8.77(\mathrm{~d}, J=4.8 \mathrm{~Hz}, 4 \mathrm{H}), 8.66(\mathrm{~d}, J=4.8$ $\mathrm{Hz}, 4 \mathrm{H}), 8.36(\mathrm{~d}, J=8.1 \mathrm{~Hz}, 4 \mathrm{H}), 8.03(\mathrm{~d}, J=8.1 \mathrm{~Hz}, 4 \mathrm{H}), 8.01(\mathrm{~d}, J=8.1 \mathrm{~Hz}, 4 \mathrm{H}), 7.54(\mathrm{t}$, $J=8.1 \mathrm{~Hz}, 2 \mathrm{H}),-2.63(\mathrm{~s}, 2 \mathrm{H})$. UV-vis $\left(\mathrm{CH}_{2} \mathrm{Cl}_{2}\right), \lambda_{\max } \mathrm{nm}(\log \varepsilon): 419(5.65), 514(4.31)$, 547(3.67), 589(3.82), 644(3.20). HRMS-EI ([M] $\left.{ }^{+}\right)$: calcd for $\mathrm{C}_{46} \mathrm{H}_{24} \mathrm{Br}_{4} \mathrm{~F}_{6} \mathrm{~N}_{4}$ 1061.8639; found: 1061.8623 with an isotope distribution pattern that is the same as the calculated one.

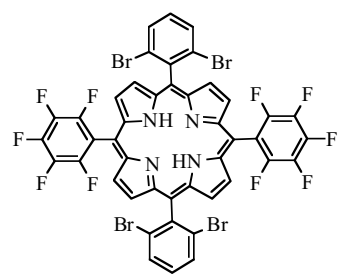

5,15-Bis(2,6-dibromophenyl)-10,20-bis(2,3,4,5,6-pentafluorophenyl)porphyrin $1 \mathrm{~d}$. Purple solid. Yield: $19 \%$. ${ }^{1} \mathrm{H}$ NMR $\left(300 \mathrm{MHz}, \mathrm{CDCl}_{3}\right): \delta 8.78(\mathrm{~d}, J=4.8 \mathrm{~Hz}, 4 \mathrm{H}), 8.73(\mathrm{~d}, J$ $=4.8 \mathrm{~Hz}, 4 \mathrm{H}), 8.04(\mathrm{~d}, J=8.1 \mathrm{~Hz}, 4 \mathrm{H}), 7.57(\mathrm{t}, J=8.1 \mathrm{~Hz}, 2 \mathrm{H}),-2.68(\mathrm{~s}, 2 \mathrm{H})$. UV-vis $\left(\mathrm{CH}_{2} \mathrm{Cl}_{2}\right), \lambda_{\max } \mathrm{nm}(\log \varepsilon): 416(5.62), 511(4.40), 547(3.72), 588(4.09), 643(3.35)$. HRMSMALDI $\left([\mathrm{M}+\mathrm{H}]^{+}\right)$: calcd for $\mathrm{C}_{44} \mathrm{H}_{17} \mathrm{Br}_{4} \mathrm{~F}_{10} \mathrm{~N}_{4} 1106.8022$; found: 1106.8009 with an isotope distribution pattern that is the same as the calculated one.

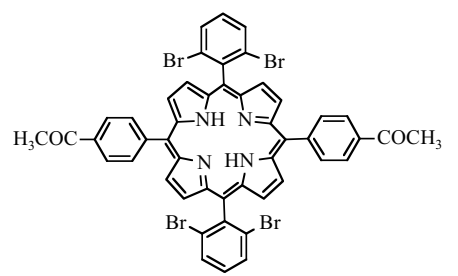

5,15-Bis(2,6-dibromophenyl)-10,20-bis(4-acetylphenyl)porphyrin 1e. Purple solid. Yield: 45\%. ${ }^{1} \mathrm{H}$ NMR (300 MHz, $\left.\mathrm{CDCl}_{3}\right): \delta 8.79(\mathrm{~d}, J=4.8 \mathrm{~Hz}, 4 \mathrm{H}), 8.66(\mathrm{~d}, J=4.8 \mathrm{~Hz}, 4 \mathrm{H}), 8.35$ (s, 8H), $8.02(\mathrm{~d}, J=8.1 \mathrm{~Hz}, 4 \mathrm{H}), 7.54(\mathrm{t}, J=8.1 \mathrm{~Hz}, 2 \mathrm{H}), 2.89(\mathrm{~s}, 6 \mathrm{H}),-2.61$ (s, 2H). 
UV-vis $\left(\mathrm{CH}_{2} \mathrm{Cl}_{2}\right), \lambda_{\max } \mathrm{nm}(\log \varepsilon): 421(5.59), 516(4.37), 549(3.79), 591(3.87), 646(3.44)$. HRMS-MALDI $\left([\mathrm{M}+\mathrm{H}]^{+}\right)$: calcd for $\mathrm{C}_{48} \mathrm{H}_{31} \mathrm{Br}_{4} \mathrm{~N}_{4} \mathrm{O}_{2} 1010.9175$ found: 1010.9171 with an isotope distribution pattern that is the same as the calculated one.

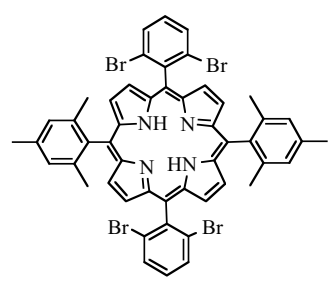

5,15-Bis(2,6-dibromophenyl)-10,20-dimesitylporphyrin 1f. Purple solid. Yield: $41 \%{ }^{1} \mathrm{H}$ NMR $\left(300 \mathrm{MHz}, \mathrm{CDCl}_{3}\right): \delta 8.66(\mathrm{~d}, J=4.8 \mathrm{~Hz}, 4 \mathrm{H}), 8.53(\mathrm{~d}, J=4.8 \mathrm{~Hz}, 4 \mathrm{H}), 8.00(\mathrm{~d}, J=$ $8.1 \mathrm{~Hz}, 4 \mathrm{H}), 7.50(\mathrm{t}, J=8.1 \mathrm{~Hz}, 2 \mathrm{H}), 7.25(\mathrm{~s}, 4 \mathrm{H}), 2.60(\mathrm{~s}, 6 \mathrm{H}), 1.84(\mathrm{~s}, 12 \mathrm{H}),-2.49(\mathrm{~s}, 2 \mathrm{H})$. UV-vis $\left(\mathrm{CH}_{2} \mathrm{Cl}_{2}\right), \lambda_{\max } \mathrm{nm}(\log \varepsilon): 419(5.75), 515(4.39), 547(3.79), 591(3.90), 647(3.46)$. HRMS-MALDI $\left([\mathrm{M}+\mathrm{H}]^{+}\right)$: calcd for $\mathrm{C}_{50} \mathrm{H}_{39} \mathrm{Br}_{4} \mathrm{~N}_{4}$ 1010.9903; found: 1010.9907 with an isotope distribution pattern that is the same as the calculated one.

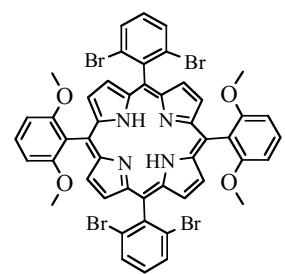

5,15-Bis(2,6-dibromophenyl)-10,20-bis(2,6-dimethoxyphenyl)porphyrin 1g. Purple solid. Yield: 69\%. ${ }^{1} \mathrm{H}$ NMR $\left(300 \mathrm{MHz}, \mathrm{CDCl}_{3}\right): \delta 8.73(\mathrm{~d}, J=4.8 \mathrm{~Hz}, 4 \mathrm{H}), 8.53(\mathrm{~d}, J=4.8 \mathrm{~Hz}$, 4H), $7.98(\mathrm{~d}, J=8.1 \mathrm{~Hz}, 4 \mathrm{H}), 7.69(\mathrm{t}, J=8.1 \mathrm{~Hz}, 2 \mathrm{H}), 7.48(\mathrm{t}, J=8.1 \mathrm{~Hz}, 2 \mathrm{H}), 6.97(\mathrm{~d}, J=$ $8.7 \mathrm{~Hz}, 4 \mathrm{H}), 3.51(\mathrm{~s}, 12 \mathrm{H}),-2.49(\mathrm{~s}, 2 \mathrm{H})$. UV-vis $\left(\mathrm{CH}_{2} \mathrm{Cl}_{2}\right), \lambda_{\max } \mathrm{nm}(\log \varepsilon): 420(5.79)$, 515(4.45), 545(3.72), 590(3.95), 644(3.26). HRMS-MALDI $\left([\mathrm{M}+\mathrm{H}]^{+}\right)$: calcd for $\mathrm{C}_{48} \mathrm{H}_{35} \mathrm{Br}_{4} \mathrm{~N}_{4} \mathrm{O}_{4} 1046.9386$; found: 1046.9434 with an isotope distribution pattern that is the same as the calculated one.

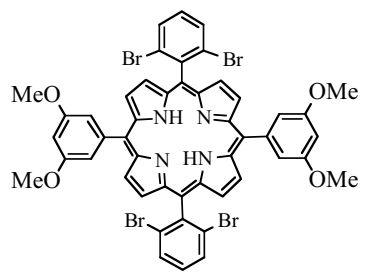

5,15-Bis(2,6-dibromophenyl)-10,20-bis(3,5-dimethoxyphenyl)porphyrin $\mathbf{1 h}$. Purple solid. Yield: 55\%. ${ }^{1} \mathrm{H}$ NMR $\left(300 \mathrm{MHz}, \mathrm{CDCl}_{3}\right): \delta 8.92(\mathrm{~d}, J=4.8 \mathrm{~Hz}, 4 \mathrm{H}), 8.61(\mathrm{~d}, J=4.8 \mathrm{~Hz}$, $4 \mathrm{H}), 8.01(\mathrm{~d}, J=8.1 \mathrm{~Hz}, 4 \mathrm{H}), 7.52(\mathrm{t}, J=8.1 \mathrm{~Hz}, 2 \mathrm{H}), 7.40(\mathrm{t}, J=2.4 \mathrm{~Hz}, 4 \mathrm{H}), 6.87(\mathrm{~d}, J=$ $2.4 \mathrm{~Hz}, 2 \mathrm{H}), 3.94(\mathrm{~s}, 12 \mathrm{H}),-2.65(\mathrm{~s}, 2 \mathrm{H})$. UV-vis $\left(\mathrm{CH}_{2} \mathrm{Cl}_{2}\right), \lambda_{\max } \mathrm{nm}(\log \varepsilon): 421(5.79)$, 515(4.44), 548(3.80), 590(3.96), 645(3.50). HRMS-MALDI $\left([\mathrm{M}+\mathrm{H}]^{+}\right)$: calcd for $\mathrm{C}_{48} \mathrm{H}_{35} \mathrm{Br}_{4} \mathrm{~N}_{4} \mathrm{O}_{4} 1046.9386$; found: 1046.9423 with an isotope distribution pattern that is the same as the calculated one. 


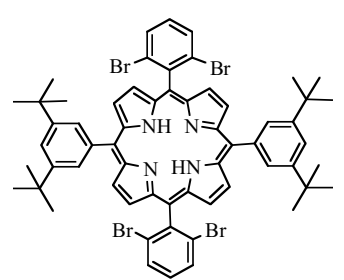

5,15-Bis(2,6-dibromophenyl)-10,20-bis[3,5-di(tert-butyl)phenyl]porphyrin 1i. Purple solid. Yield: 69\%. ${ }^{1} \mathrm{H}$ NMR (300 MHz, $\left.\mathrm{CDCl}_{3}\right): \delta 8.90(\mathrm{~d}, J=4.8 \mathrm{~Hz}, 4 \mathrm{H}), 8.65$ (d, $J=4.8$ $\mathrm{Hz}, 4 \mathrm{H}), 8.11(\mathrm{~d}, J=1.5 \mathrm{~Hz}, 4 \mathrm{H}), 8.01(\mathrm{~d}, J=8.1 \mathrm{~Hz}, 4 \mathrm{H}), 7.79(\mathrm{t}, J=1.8 \mathrm{~Hz}, 2 \mathrm{H}), 7.51(\mathrm{t}$, $J=8.1 \mathrm{~Hz}, 2 \mathrm{H}), 1.53(\mathrm{~s}, 36 \mathrm{H}),-2.52(\mathrm{~s}, 2 \mathrm{H}) .{ }^{13} \mathrm{C} \mathrm{NMR}\left(75 \mathrm{MHz}, \mathrm{CDCl}_{3}\right): \delta 148.8,143.6$, $140.6,132.3,131.4,131.0,129.9,129.3,128.5,121.6,121.1,118.1,35.1,31.7$. UV-vis $\left(\mathrm{CH}_{2} \mathrm{Cl}_{2}\right), \lambda_{\max } \mathrm{nm}(\log \varepsilon): 421(5.69), 516(4.30), 551(3.79), 592(3.79), 648(3.50)$. HRMSMALDI $\left([\mathrm{M}+\mathrm{H}]^{+}\right)$: calcd for $\mathrm{C}_{60} \mathrm{H}_{59} \mathrm{Br}_{4} \mathrm{~N}_{4}$ 1151.1468; found: 1151.1459 with an isotope distribution pattern that is the same as the calculated one.

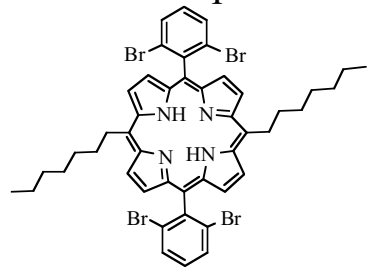

5,15-Bis(2,6-dibromophenyl)-10,20-bisheptylporphyrin 1j. Purple solid. Yield: $53 \%$. ${ }^{1} \mathrm{H}$ NMR (300 MHz, CDCl $)$ ): $\delta 9.40(\mathrm{~d}, J=4.8 \mathrm{~Hz}, 4 \mathrm{H}), 8.66(\mathrm{~d}, J=4.8 \mathrm{~Hz}, 4 \mathrm{H}), 8.03(\mathrm{~d}, J=$ $8.1 \mathrm{~Hz}, 4 \mathrm{H}), 7.54(\mathrm{t}, J=8.1 \mathrm{~Hz}, 2 \mathrm{H}), 4.89(\mathrm{t}, J=7.8 \mathrm{~Hz}, 4 \mathrm{H}), 2.54(\mathrm{~m}, 4 \mathrm{H}), 1.81(\mathrm{~m}, 4 \mathrm{H})$, 1.52(m, $4 \mathrm{H}), 1.33(\mathrm{~m}, 8 \mathrm{H}), 0.90(\mathrm{~m}, 6 \mathrm{H}),-2.45(\mathrm{~s}, 2 \mathrm{H})$. UV-vis $\left(\mathrm{CH}_{2} \mathrm{Cl}_{2}\right), \lambda_{\max } \mathrm{nm}(\log \varepsilon)$ : 419(5.61), 518(4.31), 553(3.92), 596(3.80), 654(3.74). HRMS-MALDI ([M+H] $\left.]^{+}\right)$: calcd for $\mathrm{C}_{46} \mathrm{H}_{47} \mathrm{Br}_{4} \mathrm{~N}_{4}, 971.0529$; found: 971.0510 with an isotope distribution pattern that is the same as the calculated one.

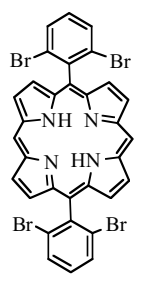

5,15-Bis(2,6-dibromophenyl)porphyrin 1k. A mixture of dipyrromethane $(0.146 \mathrm{~g}, 1$ mmol), 2,6-dibromobenzaldehyde $(0.264 \mathrm{~g}, 1 \mathrm{mmol})$ and molecular sieves $(4 \mathrm{~A}, 1.0 \mathrm{~g})$ in chloroform $(100 \mathrm{ml})$ was purged with nitrogen for $10 \mathrm{~min}$. Boron trifluoride diethyl etherate $(0.1 \mathrm{~mL})$ was added dropwise via a syringe and the flask was wrapped with aluminum foil to shield it from light. The solution was stirred under a nitrogen atmosphere at room temperature for $16 \mathrm{~h}$, and 2,3-dichloro-5,6-dicyano-1,4-benzoquinone (DDQ) (0.287 g, 1.2 mmol) was added as powder at one time. After $30 \mathrm{~min}, 1 \mathrm{~mL}$ of triethylamine was added. The reaction solution was then directly poured on the top of a silica gel column that was packed with dichloromethane. The column was eluted with dichloromethane. The fractions containing product were collected and concentrated on a rotary evaporator. The residue was washed several times with hexanes to afford the title compound as a purple solid. Yield: $0.055 \mathrm{~g}(14 \%) .{ }^{1} \mathrm{H}$ NMR (300 MHz, $\left.\mathrm{CDCl}_{3}\right): \delta 10.26(\mathrm{~s}, 2 \mathrm{H}), 9.36(\mathrm{~d}, J=4.8 \mathrm{~Hz}, 4 \mathrm{H}), 8.84$ $(\mathrm{d}, J=4.8 \mathrm{~Hz}, 4 \mathrm{H}), 8.01(\mathrm{~d}, J=8.1 \mathrm{~Hz}, 4 \mathrm{H}), 7.57(\mathrm{t}, J=8.1 \mathrm{~Hz}, 2 \mathrm{H}),-3.07(\mathrm{~s}, 2 \mathrm{H})$. UV-vis 
$\left(\mathrm{CH}_{2} \mathrm{Cl}_{2}\right), \lambda_{\max } \mathrm{nm}(\log \varepsilon): 407(5.73), 502(4.44), 534(3.95), 576(3.99), 630(3.53)$. HRMSMALDI $\left([\mathrm{M}+\mathrm{H}]^{+}\right)$: calcd for $\mathrm{C}_{32} \mathrm{H}_{19} \mathrm{Br}_{4} \mathrm{~N}_{4} 774.8338$; found: 774.8476 with an isotope distribution pattern that is the same as the calculated one.

General Procedures for Amidation of Bromoporphyrin. ${ }^{3}$ The bromoporphyrin, chiral amide, $\mathrm{Pd}(\mathrm{OAc})_{2}$, Xantphos, and $\mathrm{Cs}_{2} \mathrm{CO}_{3}$ were placed in an oven-dried, resealable Schlenk tube. The tube was capped with a Teflon screwcap, evacuated, and backfilled with nitrogen. The screwcap was replaced with a rubber septum, and THF was added via syringe. The tube was purged with nitrogen for $2 \mathrm{~min}$, and then the septum was replaced with the Teflon screwcap. The tube was sealed, and its contents were heated with stirring. The resulting mixture was cooled to room temperature, taken up in ethyl acetate and concentrated in vacuo. The crude product was then purified by flash chromatography.

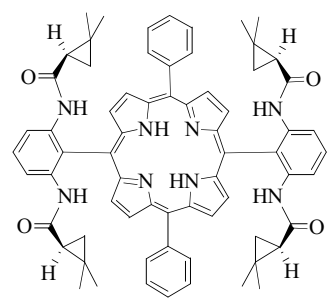

Porphyrin 3a (Table 1, entry 1). The general procedure was used to couple 5,15-bis(2,6dibromophenyl)-10,20-diphenylporphyrin $(0.093 \mathrm{~g}, 0.1 \mathrm{mmol})$ with (S)-(+)-2,2-dimethyl cyclopropanecarboxamide $(0.362 \mathrm{~g}, 3.2 \mathrm{mmol})$, using $\mathrm{Pd}(\mathrm{OAc})_{2}(0.009 \mathrm{~g}, 0.04 \mathrm{mmol})$, Xantphos $(0.046 \mathrm{~g}, 0.08 \mathrm{mmol})$, and $\mathrm{Cs}_{2} \mathrm{CO}_{3}(0.522 \mathrm{~g}, 1.6 \mathrm{mmol})$. The reaction was conducted in THF $(6 \mathrm{~mL})$ at $100{ }^{\circ} \mathrm{C}$ for $60 \mathrm{~h}$. The pure compound was isolated by flash column chromatography (silica gel, ethyl acetate:hexanes $(\mathrm{v} / \mathrm{v})=1: 2)$ as purple solids $(0.083$ g, 78\%). ${ }^{1} \mathrm{H}$ NMR $\left(300 \mathrm{MHz}, \mathrm{CDCl}_{3}\right): \delta 8.95(\mathrm{~d}, J=4.8 \mathrm{~Hz}, 4 \mathrm{H}), 8.87(\mathrm{~d}, J=4.8 \mathrm{~Hz}, 4 \mathrm{H})$, 8.44 (broad, 4H), $8.18(\mathrm{~d}, J=6.0 \mathrm{~Hz}, 4 \mathrm{H}), 7.83(\mathrm{~m}, 8 \mathrm{H}), 6.45$ (broad, $4 \mathrm{H}), 0.87(\mathrm{~s}, 12 \mathrm{H})$, 0.69 (broad, 4H), $-0.08-0.18(\mathrm{~m}, 20 \mathrm{H}),-2.65(\mathrm{~s}, 2 \mathrm{H}) .{ }^{13} \mathrm{C}$ NMR $\left(75 \mathrm{MHz}, \mathrm{CDCl}_{3}\right): \delta$ 169.6, 140.7, 139.3, 134.4, 133.6, 130.4, 128.5, 127.1, 121.4, 117.8, 28.9, 26.3, 22.4, 20.4, 18.2. UV-vis $\left(\mathrm{CH}_{2} \mathrm{Cl}_{2}\right), \lambda_{\max } \mathrm{nm}(\log \varepsilon): 420(5.33), 516(4.09), 549(3.62), 589(3.59), 644(3.33)$. HRMS-EI $\left([\mathrm{M}]^{+}\right)$: calcd for $\mathrm{C}_{68} \mathrm{H}_{66} \mathrm{~N}_{8} \mathrm{O}_{4} 1058.5207$, found 1058.5184 with an isotope distribution pattern that is the same as the calculated one.

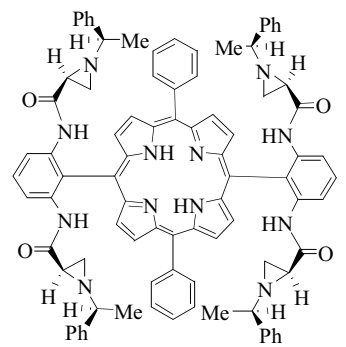

Porphyrin 3b (Table 1, entry 2). The general procedure was used to couple 5,15-bis(2,6dibromophenyl)-10,20-diphenylporphyrin $(0.023 \mathrm{~g}, 0.025 \mathrm{mmol})$ with 1 -[1'( $R)$-a-methyl benzyl]-aziridine-2 $(R)$-carboxamide $(0.076 \mathrm{~g}, 0.4 \mathrm{mmol})$, using molecular sieves $(4 \mathrm{~A}, 0.05$ g), $\mathrm{Pd}(\mathrm{OAc})_{2}(0.002 \mathrm{~g}, 0.01 \mathrm{mmol})$, Xantphos $(0.012 \mathrm{~g}, 0.02 \mathrm{mmol})$, and $\mathrm{Cs}_{2} \mathrm{CO}_{3}(0.130 \mathrm{~g}$, $0.4 \mathrm{mmol})$. The reaction was conducted in THF $(2 \mathrm{~mL})$ at $100{ }^{\circ} \mathrm{C}$ for $60 \mathrm{~h}$. The pure 
compound was isolated by flash column chromatography (silica gel, ethyl acetate:hexanes $(\mathrm{v} / \mathrm{v})=1: 1)$ as purple solids $(0.022 \mathrm{~g}, 64 \%) .{ }^{1} \mathrm{H}$ NMR $\left(300 \mathrm{MHz}, \mathrm{CDCl}_{3}\right): \delta 8.89(\mathrm{~m}, 8 \mathrm{H})$, $8.58(\mathrm{~m}, 8 \mathrm{H}), 7.93(\mathrm{t}, J=8.7 \mathrm{~Hz}, 2 \mathrm{H}), 7.55(\mathrm{t}, J=7.2 \mathrm{~Hz}, 2 \mathrm{H}), 7.34(\mathrm{t}, J=7.8 \mathrm{~Hz}, 4 \mathrm{H}), 6.75$ $(\mathrm{d}, J=7.2 \mathrm{~Hz}, 4 \mathrm{H}), 5.87(\mathrm{t}, J=7.8 \mathrm{~Hz}, 4 \mathrm{H}), 4.59(\mathrm{~m}, 16 \mathrm{H}), 1.63(\mathrm{~m}, 8 \mathrm{H}), 0.53(\mathrm{~d}, J=6.3 \mathrm{~Hz}$, $12 \mathrm{H}), 0.27(\mathrm{~m}, 8 \mathrm{H}),-2.08(\mathrm{~s}, 2 \mathrm{H}) .{ }^{13} \mathrm{C}$ NMR $\left(75 \mathrm{MHz}, \mathrm{CDCl}_{3}\right): \delta 168.7,141.5,140.6$, 139.0, 133.2, 130.9, 127.7, 126.1, 125.3, 123.8, 121.8, 120.3, 116.0, 108.7, 66.6, 39.4, 34.0, 23.5. UV-vis $\left(\mathrm{CH}_{2} \mathrm{Cl}_{2}\right), \lambda_{\max } \mathrm{nm}(\log \varepsilon)$ : 421(5.60), 514(4.35), 548(3.92), 590(3.84), 646(3.73). HRMS-MALDI ([M] $\left.]^{+}\right)$: calcd for $\mathrm{C}_{88} \mathrm{H}_{79} \mathrm{~N}_{12} \mathrm{O}_{4} 1367.6342$, found 1367.6343 with an isotope distribution pattern that is the same as the calculated one.

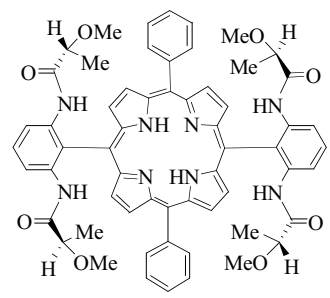

Porphyrin 3c (Table 1, entry 3 ). The general procedure was used to couple 5,15-bis(2,6dibromophenyl)-10,20-diphenylporphyrin $(0.046 \mathrm{~g}, 0.05 \mathrm{mmol})$ with $(\mathrm{R})-(+)-2$-methoxy propionamide $(0.082 \mathrm{~g}, 0.8 \mathrm{mmol})$, using molecular sieves $(4 \mathrm{~A}, 0.100 \mathrm{~g}), \mathrm{Pd}(\mathrm{OAc})_{2}(0.004 \mathrm{~g}$, $0.02 \mathrm{mmol})$, Xantphos (0.023 g, $0.04 \mathrm{mmol})$, and $\mathrm{Cs}_{2} \mathrm{CO}_{3}(0.261 \mathrm{~g}, 0.8 \mathrm{mmol})$. The reaction was conducted in THF $(4 \mathrm{~mL})$ at $100{ }^{\circ} \mathrm{C}$ for $64 \mathrm{~h}$. The pure compound was isolated by flash column chromatography (silica gel, ethyl acetate:hexanes $(\mathrm{v} / \mathrm{v})=1: 1)$ as purple solids $(0.038$ g, 75\%). ${ }^{1} \mathrm{H}$ NMR $\left(300 \mathrm{MHz}, \mathrm{CDCl}_{3}\right): \delta 8.86(\mathrm{~d}, J=4.8 \mathrm{~Hz}, 4 \mathrm{H}), 8.79(\mathrm{~d}, J=4.8 \mathrm{~Hz}, 4 \mathrm{H})$, $8.53(\mathrm{~d}, J=8.1 \mathrm{~Hz}, 4 \mathrm{H}), 8.09$ (d, $J=7.2 \mathrm{~Hz}, 4 \mathrm{H}), 7.88(\mathrm{t}, J=8.1 \mathrm{~Hz}, 2 \mathrm{H}), 7.77(\mathrm{~m}, 10 \mathrm{H})$, $3.03(\mathrm{q}, J=6.6 \mathrm{~Hz}, 4 \mathrm{H}), 1.22(\mathrm{~s}, 12 \mathrm{H}), 0.62(\mathrm{~d}, J=6.6 \mathrm{~Hz}, 12 \mathrm{H}),-2.56(\mathrm{~s}, 2 \mathrm{H}) .{ }^{13} \mathrm{C}$ NMR (75 MHz, $\left.\mathrm{CDCl}_{3}\right): \delta 171.1,140.9,138.4,134.4,130.7,128.3,127.1,122.4,121.2,117.2$, 108.2, 78.0, 55.8, 17.7. UV-vis $\left(\mathrm{CH}_{2} \mathrm{Cl}_{2}\right), \lambda_{\max } \mathrm{nm}(\log \varepsilon): 419(5.51), 514(4.24), 547(3.76)$, 589(3.72), 644(3.49). HRMS-MALDI ([M] $\left.]^{+}\right)$: calcd for $\mathrm{C}_{60} \mathrm{H}_{59} \mathrm{~N}_{8} \mathrm{O}_{8}$ 1019.4450, found 1019.4462 with an isotope distribution pattern that is the same as the calculated one.

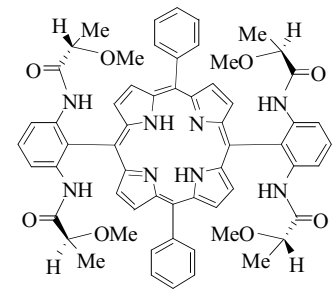

Porphyrin 3d (Table 1, entry 4). The general procedure was used to couple 5,15-bis(2,6dibromophenyl)-10,20-diphenylporphyrin $(0.046 \mathrm{~g}, 0.05 \mathrm{mmol})$ with (S)-(-)-2-methoxy propionamide $(0.082 \mathrm{~g}, 0.8 \mathrm{mmol})$, using molecular sieves $(4 \mathrm{~A}, 0.1 \mathrm{~g}), \mathrm{Pd}(\mathrm{OAc})_{2}(0.004 \mathrm{~g}$, $0.02 \mathrm{mmol})$, Xantphos (0.023 g, $0.04 \mathrm{mmol})$, and $\mathrm{Cs}_{2} \mathrm{CO}_{3}(0.261 \mathrm{~g}, 0.8 \mathrm{mmol})$. The reaction was conducted in THF $(4 \mathrm{~mL})$ at $80{ }^{\circ} \mathrm{C}$ for $62 \mathrm{~h}$. The pure compound was isolated by flash column chromatography (silica gel, ethyl acetate:hexanes $(\mathrm{v} / \mathrm{v})=1: 1)$ as purple solids $(0.036$ g, 71\%). ${ }^{1} \mathrm{H}$ NMR (300 MHz, $\left.\mathrm{CDCl}_{3}\right): \delta 8.86(\mathrm{~d}, J=4.8 \mathrm{~Hz}, 4 \mathrm{H}), 8.79(\mathrm{~d}, J=4.8 \mathrm{~Hz}, 4 \mathrm{H})$, $8.53(\mathrm{~d}, J=8.1 \mathrm{~Hz}, 4 \mathrm{H}), 8.09(\mathrm{~d}, J=6.9 \mathrm{~Hz}, 4 \mathrm{H}), 7.88(\mathrm{t}, J=8.1 \mathrm{~Hz}, 2 \mathrm{H}), 7.77(\mathrm{~m}, 10 \mathrm{H})$, $3.03(\mathrm{q}, J=7.2 \mathrm{~Hz}, 4 \mathrm{H}), 1.22(\mathrm{~s}, 12 \mathrm{H}), 0.62(\mathrm{~d}, J=6.6 \mathrm{~Hz}, 12 \mathrm{H}),-2.57(\mathrm{~s}, 2 \mathrm{H}) .{ }^{13} \mathrm{C}$ NMR 
(75 MHz, $\left.\mathrm{CDCl}_{3}\right): \delta 171.1,140.9,138.4,134.4,130.7,128.3,127.1,122.3,121.2,117.1$, 108.1, 78.0, 55.8, 17.7. UV-vis $\left(\mathrm{CH}_{2} \mathrm{Cl}_{2}\right), \lambda_{\max } \mathrm{nm}(\log \varepsilon)$ : 419(5.50), 514(4.23), 547(3.74), 587(3.70), 644(3.44). HRMS-MALDI ([M] $\left.]^{+}\right)$: calcd for $\mathrm{C}_{60} \mathrm{H}_{59} \mathrm{~N}_{8} \mathrm{O}_{8}$ 1019.4450, found 1019.4497 with an isotope distribution pattern that is the same as the calculated one.

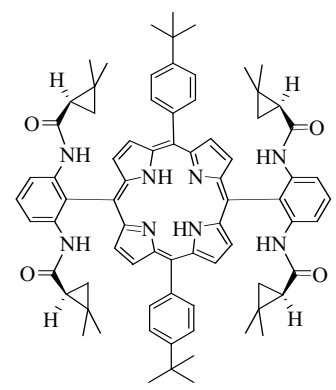

Porphyrin 3e (Table 1, entry 5). The general procedure was used to couple 5,15-bis(2,6dibromophenyl)-10,20-bis[4-(tert-butyl)phenyl]porphyrin $(0.078 \mathrm{~g}, 0.075 \mathrm{mmol})$ with (S)(+)-2,2-dimethylcyclopropanecarboxamide $(0.136 \mathrm{~g}, 1.2 \mathrm{mmol})$, using $\mathrm{Pd}(\mathrm{OAc})_{2}(0.007 \mathrm{~g}$, $0.03 \mathrm{mmol})$, Xantphos (0.035 g, $0.06 \mathrm{mmol})$, and $\mathrm{Cs}_{2} \mathrm{CO}_{3}(0.391 \mathrm{~g}, 1.2 \mathrm{mmol})$. The reaction was conducted in THF $(6 \mathrm{~mL})$ at $100{ }^{\circ} \mathrm{C}$ for $40 \mathrm{~h}$. The pure compound was isolated by flash column chromatography (silica gel, ethyl acetate:hexanes $(\mathrm{v} / \mathrm{v})=1: 4)$ as purple solids $(0.076$ g, 86\%). ${ }^{1} \mathrm{H}$ NMR $\left(300 \mathrm{MHz}, \mathrm{CDCl}_{3}\right): \delta 8.99(\mathrm{~d}, J=4.8 \mathrm{~Hz}, 4 \mathrm{H}), 8.85(\mathrm{~d}, J=4.8 \mathrm{~Hz}, 4 \mathrm{H})$, 8.45 (broad, 4H), $8.10(\mathrm{~d}, J=8.1 \mathrm{~Hz}, 4 \mathrm{H}), 7.81(\mathrm{~m}, 6 \mathrm{H}), 6.46$ (broad, $4 \mathrm{H}), 1.61(\mathrm{~s}, 18 \mathrm{H})$, 0.87 (s, 12H), 0.67 (broad, 4H), -0.11-0.17 (m, 20H), -2.63 (s, 2H) ${ }^{13} \mathrm{C}$ NMR (75 MHz, $\left.\mathrm{CDCl}_{3}\right): \delta 169.7,151.4,139.3,137.7,134.0,130.3,124.1,121.68,117.5,35.0,31.6,29.1$, 26.3, 22.4, 20.4, 18.2. UV-vis $\left(\mathrm{CH}_{2} \mathrm{Cl}_{2}\right), \lambda_{\max } \mathrm{nm}(\log \varepsilon): 421(5.38), 516(4.10), 552(3.69)$, 591(3.59), 648(3.49). HRMS-EI ([M] $\left.]^{+}\right)$: calcd for $\mathrm{C}_{76} \mathrm{H}_{82} \mathrm{~N}_{8} \mathrm{O}_{4} 1170.6459$, found 1170.6451 with an isotope distribution pattern that is the same as the calculated one.

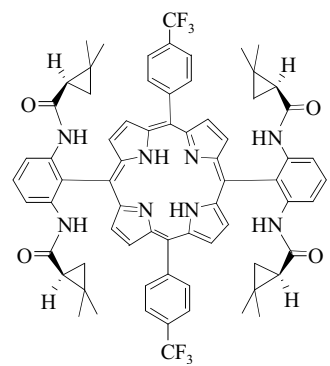

Porphyrin 3f (Table 1, entry 6). The general procedure was used to couple 5,15-bis(2,6dibromophenyl)-10,20-bis(4-trifluoromethylphenyl)porphyrin (0.053 g, $0.05 \mathrm{mmol}$ ) with (S)(+)-2,2-dimethylcyclopropanecarboxamide $(0.184 \mathrm{~g}, 1.6 \mathrm{mmol})$, using $\mathrm{Pd}(\mathrm{OAc})_{2}(0.004 \mathrm{~g}$, $0.02 \mathrm{mmol})$, Xantphos (0.023 g, $0.04 \mathrm{mmol})$, and $\mathrm{Cs}_{2} \mathrm{CO}_{3}(0.261 \mathrm{~g}, 0.8 \mathrm{mmol})$. The reaction was conducted in THF $(4 \mathrm{~mL})$ at $100{ }^{\circ} \mathrm{C}$ for $60 \mathrm{~h}$. The pure compound was isolated by flash column chromatography (silica gel, ethyl acetate:hexanes $(\mathrm{v} / \mathrm{v})=1: 2)$ as purple solids $(0.046$ g, 77\%). ${ }^{1} \mathrm{H}$ NMR $\left(300 \mathrm{MHz}, \mathrm{CDCl}_{3}\right): \delta 8.89(\mathrm{~m}, 8 \mathrm{H}), 8.41$ (broad, $\left.4 \mathrm{H}\right), 8.31(\mathrm{~d}, J=8.1 \mathrm{~Hz}$, $4 \mathrm{H}), 8.08(\mathrm{~d}, J=8.1 \mathrm{~Hz}, 4 \mathrm{H}), 7.83(\mathrm{t}, J=8.1 \mathrm{~Hz}, 2 \mathrm{H}), 6.41$ (broad, 4H), $0.85(\mathrm{~s}, 12 \mathrm{H}), 0.69$ (broad, $4 \mathrm{H}),-0.07-0.19(\mathrm{~m}, 20 \mathrm{H}),-2.68(\mathrm{~s}, 2 \mathrm{H}) .{ }^{13} \mathrm{C}$ NMR $\left(75 \mathrm{MHz}, \mathrm{CDCl}_{3}\right): \delta 189.8$, $170.2,144.4,139.3,134.5,130.6,124.2,119.6,29.0,26.3,22.5,20.4,18.2$. UV-vis $\left(\mathrm{CH}_{2} \mathrm{Cl}_{2}\right), \lambda_{\max } \mathrm{nm}(\log \varepsilon): 420(5.53), 514(4.33), 547(3.77), 588(3.82), 643(3.43)$. HRMS- 
MALDI $\left([\mathrm{M}+\mathrm{H}]^{+}\right)$: calcd for $\mathrm{C}_{70} \mathrm{H}_{65} \mathrm{~F}_{6} \mathrm{~N}_{8} \mathrm{O}_{8} 1195.5027$, found 1195.5085 with an isotope distribution pattern that is the same as the calculated one.

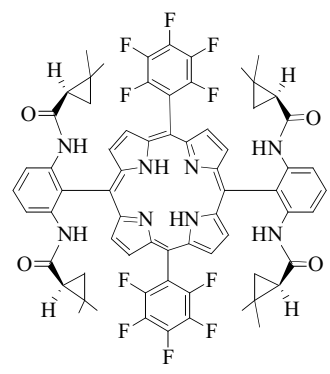

Porphyrin 3g (Table 1, entry 7). The general procedure was used to couple 5,15-bis(2,6dibromophenyl)-10,20-bis(2,3,4,5,6-pentafluorophenyl)porphyrin (0.028 g, $0.025 \mathrm{mmol})$ with (S)-(+)-2,2-dimethylcyclopropanecarboxamide $(0.091 \mathrm{~g}, 0.8 \mathrm{mmol})$, using $\mathrm{Pd}(\mathrm{OAc})_{2}$ (0.002 g, $0.01 \mathrm{mmol})$, Xantphos $(0.012 \mathrm{~g}, 0.02 \mathrm{mmol})$, and $\mathrm{Cs}_{2} \mathrm{CO}_{3}(0.130 \mathrm{~g}, 0.4 \mathrm{mmol})$. The reaction was conducted in THF $(2 \mathrm{~mL})$ at $100{ }^{\circ} \mathrm{C}$ for $60 \mathrm{~h}$. The pure compound was isolated by flash column chromatography (silica gel, ethyl acetate:hexanes $(\mathrm{v} / \mathrm{v})=1: 4)$ as purple solids $(0.015 \mathrm{~g}, 46 \%) .{ }^{1} \mathrm{H}$ NMR $\left(300 \mathrm{MHz}, \mathrm{CDCl}_{3}\right): \delta 8.99(\mathrm{~d}, J=4.8 \mathrm{~Hz}, 4 \mathrm{H}), 8.91(\mathrm{~d}, J=$ $4.8 \mathrm{~Hz}, 4 \mathrm{H}), 8.36$ (broad, 4H), $7.84(\mathrm{t}, J=8.1 \mathrm{~Hz}, 2 \mathrm{H}), 6.37$ (broad, 4H), $0.81(\mathrm{~s}, 12 \mathrm{H}), 0.69$ (broad, $4 \mathrm{H}),-0.02-0.14(\mathrm{~m}, 20 \mathrm{H}),-2.71(\mathrm{~s}, 2 \mathrm{H}) .{ }^{13} \mathrm{C}$ NMR $\left(75 \mathrm{MHz}, \mathrm{CDCl}_{3}\right): \delta 169.8$, $139.1,130.8,118.7,110.8,103.5,28.8,26.1,22.5,20.4,18.1$. UV-vis $\left(\mathrm{CH}_{2} \mathrm{Cl}_{2}\right), \lambda_{\max } \mathrm{nm}$ $(\log \varepsilon)$ : 419(5.54), 511(4.46), 544(3.64), 585(3.98), 639(3.15). HRMS-MALDI $\left([\mathrm{M}+\mathrm{H}]^{+}\right)$: calcd for $\mathrm{C}_{68} \mathrm{H}_{57} \mathrm{~F}_{10} \mathrm{~N}_{8} \mathrm{O}_{4} 1239.4338$, found 1239.4335 with an isotope distribution pattern that is the same as the calculated one.

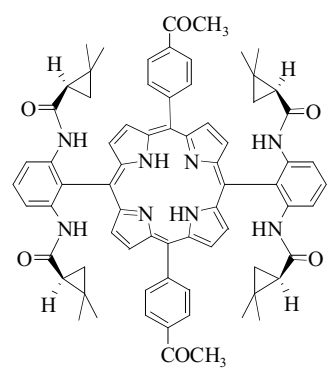

Porphyrin 3h (Table 1, entry 8 ). The general procedure was used to couple 5,15-bis(2,6dibromophenyl)-10,20-bis(4-acetylphenyl)porphyrin (0.051 g, $0.05 \mathrm{mmol})$ with (S)-(+)-2,2dimethylcyclopropanecarboxamide $(0.184 \mathrm{~g}, 1.6 \mathrm{mmol})$, using $\mathrm{Pd}(\mathrm{OAc})_{2}(0.004 \mathrm{~g}, 0.02$ mmol), Xantphos $(0.023 \mathrm{~g}, 0.04 \mathrm{mmol})$, and $\mathrm{Cs}_{2} \mathrm{CO}_{3}(0.261 \mathrm{~g}, 0.8 \mathrm{mmol})$. The reaction was conducted in THF $(4 \mathrm{~mL})$ at $100{ }^{\circ} \mathrm{C}$ for $60 \mathrm{~h}$. The pure compound was isolated by flash column chromatography (silica gel, ethyl acetate:methylene chloride $(\mathrm{v} / \mathrm{v})=1: 3)$ as purple solids $(0.038 \mathrm{~g}, 66 \%) .{ }^{1} \mathrm{H}$ NMR $\left(300 \mathrm{MHz}, \mathrm{CDCl}_{3}\right): \delta 8.92(\mathrm{~m}, 8 \mathrm{H}), 8.30-8.42(\mathrm{~m}, 12 \mathrm{H}), 7.83$ $(\mathrm{t}, J=8.1 \mathrm{~Hz}, 2 \mathrm{H}), 6.46$ (broad, 4H), 2.89 (s, 6H), 0.71-0.88 (m, 16H), -0.04-0.20 (m, $20 \mathrm{H}),-2.63(\mathrm{~s}, 2 \mathrm{H}) .{ }^{13} \mathrm{C}$ NMR $\left(75 \mathrm{MHz}, \mathrm{CDCl}_{3}\right): \delta 197.9,171.0,169.6,145.5,139.2,136.8$, 134.6, 130.5, 127.0, 118.0, 28.9, 27.0, 26.3, 22.4, 20.4, 18.2. UV-vis $\left(\mathrm{CH}_{2} \mathrm{Cl}_{2}\right), \lambda_{\max } \mathrm{nm}(\log$ $\varepsilon)$ : 412(5.55), 516(4.32), 550(3.82), 589(3.79), 644(3.40). HRMS-MALDI ([M+H] $\left.]^{+}\right)$: calcd for $\mathrm{C}_{72} \mathrm{H}_{71} \mathrm{~N}_{8} \mathrm{O}_{6} 1143.5491$, found 1143.5467 with an isotope distribution pattern that is the same as the calculated one. 


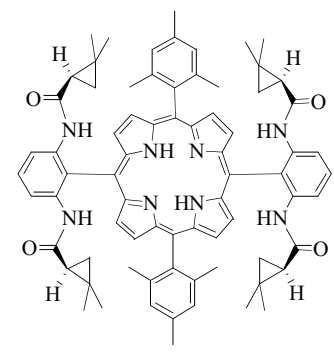

Porphyrin 3i (Table 1, entry 9). The general procedure was used to couple 5,15-bis(2,6dibromophenyl)-10,20-dimesitylporphyrin $(0.051 \mathrm{~g}, 0.05 \mathrm{mmol})$ with $(\mathrm{S})-(+)-2,2$-dimethyl cyclopropanecarboxamide $(0.181 \mathrm{~g}, 1.6 \mathrm{mmol})$, using $\mathrm{Pd}(\mathrm{OAc})_{2}(0.004 \mathrm{~g}, 0.02 \mathrm{mmol})$, Xantphos $(0.023 \mathrm{~g}, 0.04 \mathrm{mmol})$, and $\mathrm{Cs}_{2} \mathrm{CO}_{3}(0.261 \mathrm{~g}, 0.8 \mathrm{mmol})$. The reaction was conducted in THF $(6 \mathrm{~mL})$ at $100{ }^{\circ} \mathrm{C}$ for $56 \mathrm{~h}$. The pure compound was isolated by flash column chromatography (silica gel, ethyl acetate:hexanes $(\mathrm{v} / \mathrm{v})=1: 4)$ as purple solids $(0.048$ g, 84\%). ${ }^{1} \mathrm{H}$ NMR (300 MHz, $\left.\mathrm{CDCl}_{3}\right): \delta 8.80(\mathrm{~m}, 8 \mathrm{H}), 8.42$ (broad, 4H), $7.80(\mathrm{t}, J=8.1 \mathrm{~Hz}$, 2H), $7.30(\mathrm{~s}, 4 \mathrm{H}), 6.52$ (broad, 4H), $2.63(\mathrm{~s}, 6 \mathrm{H}), 1.82(\mathrm{~s}, 12 \mathrm{H}), 0.86(\mathrm{~s}, 12 \mathrm{H}), 0.68$ (broad, $4 \mathrm{H}),-0.06-0.20(\mathrm{~m}, 20 \mathrm{H}),-2.54(\mathrm{~s}, 2 \mathrm{H}) .{ }^{13} \mathrm{C} \mathrm{NMR}\left(75 \mathrm{MHz}, \mathrm{CDCl}_{3}\right): \delta 169.6,147.3,139.2$, 138.9, 138.6, 136.9, 130.4, 128.2, 119.9, 117.6, 28.9, 26.4, 22.4, 21.7, 21.5, 20.5, 18.2. UV-vis $\left(\mathrm{CH}_{2} \mathrm{Cl}_{2}\right), \lambda_{\max } \mathrm{nm}(\log \varepsilon)$ : 421(5.42), 515(4.19), 549(3.68), 590(3.68), 645(3.48). HRMS-EI $\left([\mathrm{M}]^{+}\right)$: calcd for $\mathrm{C}_{74} \mathrm{H}_{78} \mathrm{~N}_{8} \mathrm{O}_{4} 1142.6146$, found 1142.6115 with an isotope distribution pattern that is the same as the calculated one.

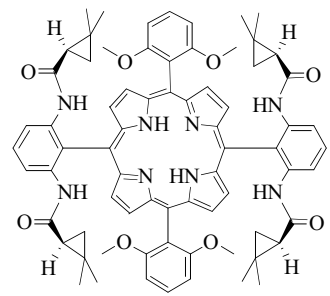

Porphyrin 3j (Table 1, entry 10). The general procedure was used to couple 5,15-bis(2,6dibromophenyl)-10,20-bis(2,6-dimethoxyphenyl)porphyrin $(0.105 \mathrm{~g}, 0.1 \mathrm{mmol})$ with $(\mathrm{S})-(+)-$ 2,2-dimethylcyclopropanecarboxamide $(0.362 \mathrm{~g}, 3.2 \mathrm{mmol})$, using $\mathrm{Pd}(\mathrm{OAc})_{2}(0.009 \mathrm{~g}, 0.04$ mmol), Xantphos (0.046 g, $0.08 \mathrm{mmol})$, and $\mathrm{Cs}_{2} \mathrm{CO}_{3}(0.522 \mathrm{~g}, 1.6 \mathrm{mmol})$. The reaction was conducted in THF $(6 \mathrm{~mL})$ at $100{ }^{\circ} \mathrm{C}$ for $60 \mathrm{~h}$. The pure compound was isolated by flash column chromatography (silica gel, ethyl acetate:hexanes $(\mathrm{v} / \mathrm{v})=1: 2)$ as purple solids $(0.069$ g, 59\%). ${ }^{1} \mathrm{H}$ NMR (300 MHz, $\left.\mathrm{CDCl}_{3}\right): \delta 8.87(\mathrm{~d}, J=4.8 \mathrm{~Hz}, 4 \mathrm{H}), 8.79(\mathrm{~d}, J=4.8 \mathrm{~Hz}, 4 \mathrm{H})$, 8.47 (broad, 4H), $7.81(\mathrm{t}, J=8.7 \mathrm{~Hz}, 4 \mathrm{H}), 7.06(\mathrm{~d}, J=8.4 \mathrm{~Hz}, 4 \mathrm{H}), 6.58(\mathrm{broad}, 4 \mathrm{H}), 3.55(\mathrm{~s}$, $12 \mathrm{H}), 0.88(\mathrm{~s}, 12 \mathrm{H}), 0.65$ (broad, $4 \mathrm{H}), 0.04-0.21(\mathrm{~m}, 20 \mathrm{H}),-2.47(\mathrm{~s}, 2 \mathrm{H}) .{ }^{13} \mathrm{C}$ NMR $(75$ $\left.\mathrm{MHz}_{\mathrm{CDCl}}\right): \delta 169.6,160.2,139.2,130.9,130.1,118.2,117.0,113.6,107.1,104.1,55.9$, 29.0, 26.2, 22.2, 20.1, 18.2. UV-vis $\left(\mathrm{CH}_{2} \mathrm{Cl}_{2}\right), \lambda_{\max } \mathrm{nm}(\log \varepsilon): 412(5.50), 514(4.28)$, 547(3.67), 589(3.77), 643(3.38). HRMS-MALDI $\left([\mathrm{M}+\mathrm{H}]^{+}\right)$: calcd for $\mathrm{C}_{72} \mathrm{H}_{75} \mathrm{~N}_{8} \mathrm{O}_{8}$ 1179.5702 , found 1179.5758 with an isotope distribution pattern that is the same as the calculated one. 


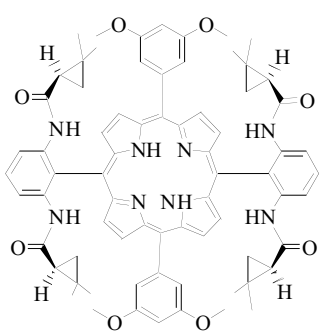

Porphyrin 3k (Table 1, entry 11). The general procedure was used to couple 5,15-bis(2,6dibromophenyl)-10,20-bis(3,5-dimethoxyphenyl)porphyrin $(0.053 \mathrm{~g}, 0.05 \mathrm{mmol})$ with (S)(+)-2,2-dimethylcyclopropanecarboxamide $(0.181 \mathrm{~g}, 1.6 \mathrm{mmol})$, using $\mathrm{Pd}(\mathrm{OAc})_{2}(0.004 \mathrm{~g}$, $0.02 \mathrm{mmol})$, Xantphos (0.023 g, $0.04 \mathrm{mmol})$, and $\mathrm{Cs}_{2} \mathrm{CO}_{3}(0.261 \mathrm{~g}, 0.8 \mathrm{mmol})$. The reaction was conducted in THF $(4 \mathrm{~mL})$ at $100{ }^{\circ} \mathrm{C}$ for $48 \mathrm{~h}$. The pure compound was isolated by flash column chromatography (silica gel, ethyl acetate:hexanes $(\mathrm{v} / \mathrm{v})=1: 1)$ as purple solids $(0.052$ g, 88\%). ${ }^{1} \mathrm{H}$ NMR (300 MHz, $\left.\mathrm{CDCl}_{3}\right): \delta 9.04(\mathrm{~d}, J=4.8 \mathrm{~Hz}, 4 \mathrm{H}), 8.84(\mathrm{~d}, J=4.8 \mathrm{~Hz}, 4 \mathrm{H})$, 8.44 (broad, 4H), $7.83(\mathrm{t}, J=8.7 \mathrm{~Hz}, 2 \mathrm{H}), 7.34(\mathrm{~d}, J=1.8 \mathrm{~Hz}, 4 \mathrm{H}), 6.93(\mathrm{t}, J=1.8 \mathrm{~Hz}, 2 \mathrm{H})$, 6.45 (broad, 4H), 3.98 (s, 12H), 0.88 (s, 12H), 0.69 (broad, 4H), -0.07-0.17 (m, 20H), -2.68 $(\mathrm{s}, 2 \mathrm{H}) .{ }^{13} \mathrm{C}$ NMR $\left(75 \mathrm{MHz}, \mathrm{CDCl}_{3}\right): \delta 169.6,159.1,142.5,139.3,133.7,130.4,121.0$, 117.7, 114.1, 100.1, 55.6, 29.0, 26.3, 22.4, 20.4, 18.3. UV-vis $\left(\mathrm{CH}_{2} \mathrm{Cl}_{2}\right), \lambda_{\max } \mathrm{nm}(\log \varepsilon)$ : 423(5.53), 515(4.34), 549(3.81), 589(3.85), 643(3.55). HRMS-MALDI ([M+H] $\left.{ }^{+}\right)$: calcd for $\mathrm{C}_{72} \mathrm{H}_{75} \mathrm{~N}_{8} \mathrm{O}_{8} 1179.5702$, found 1179.5736 with an isotope distribution pattern that is the same as the calculated one.

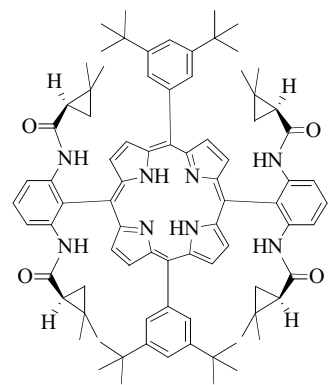

Porphyrin 31 (Table 1, entry 12). The general procedure was used to couple 5,15-bis(2,6dibromophenyl)-10,20-bis[3,5-di(tert-butyl)phenyl]porphyrin $(0.231 \mathrm{~g}, 0.2 \mathrm{mmol})$ with (S)(+)-2,2-dimethylcyclopropanecarboxamide (0.362 g, $3.2 \mathrm{mmol})$, using $\mathrm{Pd}(\mathrm{OAc})_{2}(0.018 \mathrm{~g}$, $0.08 \mathrm{mmol})$, Xantphos $(0.093 \mathrm{~g}, 0.16 \mathrm{mmol})$, and $\mathrm{Cs}_{2} \mathrm{CO}_{3}(1.045 \mathrm{~g}, 3.2 \mathrm{mmol})$. The reaction was conducted in THF $(4 \mathrm{~mL})$ at $100{ }^{\circ} \mathrm{C}$ for $48 \mathrm{~h}$. The pure compound was isolated by flash column chromatography (silica gel, ethyl acetate:hexanes $(\mathrm{v} / \mathrm{v})=1: 4)$ as purple solids $(0.217$ g, 85\%). ${ }^{1} \mathrm{H}$ NMR (300 MHz, $\left.\mathrm{CDCl}_{3}\right): \delta 8.99(\mathrm{~d}, J=4.8 \mathrm{~Hz}, 4 \mathrm{H}), 8.87(\mathrm{~d}, J=4.8 \mathrm{~Hz}, 4 \mathrm{H})$, 8.44 (broad, 4H), 8.04 (d, $J=1.5 \mathrm{~Hz}, 4 \mathrm{H}), 7.83$ (m, 4H), 6.50 (broad, 4H), 1.53 (s, 36H), $0.87(\mathrm{~s}, 12 \mathrm{H}), 0.69$ (broad, 4H), $-0.05-0.14(\mathrm{~m}, 20 \mathrm{H}),-2.34(\mathrm{~s}, 2 \mathrm{H}) .{ }^{13} \mathrm{C}$ NMR $(75 \mathrm{MHz}$, $\left.\mathrm{CDCl}_{3}\right): \delta 169.6,149.3,139.8,139.2,133.6,130.3,129.8,122.7,121.8,117.4,35.0,31.7$, 29.0, 26.3, 22.3, 20.2, 18.3. UV-vis $\left(\mathrm{CH}_{2} \mathrm{Cl}_{2}\right), \lambda_{\max } \mathrm{nm}(\log \varepsilon): 422(5.46), 517(4.17)$, 552(3.77), 591(3.66), 646(3.53). HRMS-EI ([M] $\left.]^{+}\right)$: calcd for $\mathrm{C}_{84} \mathrm{H}_{98} \mathrm{~N}_{8} \mathrm{O}_{4}$ 1282.7711, found 1282.7715 with an isotope distribution pattern that is the same as the calculated one. 


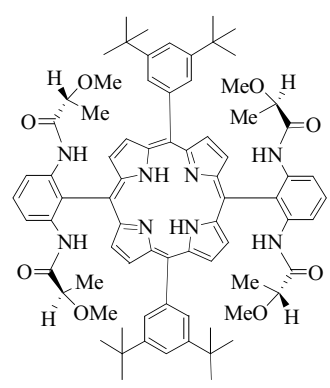

Porphyrin 3m (Table 1, entry 13). The general procedure was used to couple 5,15-bis(2,6dibromophenyl)-10,20-bis[3,5-di(tert-butyl)phenyl]porphyrin (0.058 g, $0.05 \mathrm{mmol})$ with (R)$(+)$-2-methoxypropionamide $(0.082 \mathrm{~g}, 0.8 \mathrm{mmol})$, using molecular sieves $(4 \mathrm{~A}, 0.100 \mathrm{~g})$, $\mathrm{Pd}(\mathrm{OAc})_{2}(0.004 \mathrm{~g}, 0.02 \mathrm{mmol})$, Xantphos $(0.023 \mathrm{~g}, 0.04 \mathrm{mmol})$, and $\mathrm{Cs}_{2} \mathrm{CO}_{3}(0.261 \mathrm{~g}, 0.8$ $\mathrm{mmol})$. The reaction was conducted in THF $(4 \mathrm{~mL})$ at $100{ }^{\circ} \mathrm{C}$ for $64 \mathrm{~h}$. The pure compound was isolated by flash column chromatography (silica gel, ethyl acetate:hexanes $(\mathrm{v} / \mathrm{v})=1: 2$ ) as purple solids $(0.049 \mathrm{~g}, 79 \%) .{ }^{1} \mathrm{H}$ NMR $\left(300 \mathrm{MHz}, \mathrm{CDCl}_{3}\right): \delta 8.90(\mathrm{~d}, J=4.8 \mathrm{~Hz}, 4 \mathrm{H})$, $8.79(\mathrm{~d}, J=4.8 \mathrm{~Hz}, 4 \mathrm{H}), 8.57(\mathrm{~d}, J=8.7 \mathrm{~Hz}, 4 \mathrm{H}), 7.95(\mathrm{~s}, 4 \mathrm{H}), 7.89(\mathrm{t}, J=8.7 \mathrm{~Hz}, 2 \mathrm{H}), 7.83$ $(\mathrm{s}, 6 \mathrm{H}), 3.08(\mathrm{q}, J=6.6 \mathrm{~Hz}, 4 \mathrm{H}), 1.52(\mathrm{~s}, 36 \mathrm{H}), 1.36(\mathrm{~s}, 12 \mathrm{H}), 0.66(\mathrm{~d}, J=6.6 \mathrm{~Hz}, 12 \mathrm{H})$, $-2.48(\mathrm{~s}, 2 \mathrm{H}) .{ }^{13} \mathrm{C}$ NMR $\left(75 \mathrm{MHz}, \mathrm{CDCl}_{3}\right): \delta 171.2,149.2,140.0,138.5,130.6,129.8$, $122.6,122.3,121.7,117.0,107.8,78.1,55.9,35.0,31.6,17.7$. UV-vis $\left(\mathrm{CH}_{2} \mathrm{Cl}_{2}\right), \lambda_{\max } \mathrm{nm}$ $(\log \varepsilon):$ 421(5.59), 516(4.29), 551(3.89), 592(3.76), 647(3.62). HRMS-MALDI ([M] $\left.]^{+}\right)$: calcd for $\mathrm{C}_{76} \mathrm{H}_{91} \mathrm{~N}_{8} \mathrm{O}_{8} 1243.6954$, found 1243.6894 with an isotope distribution pattern that is the same as the calculated one.

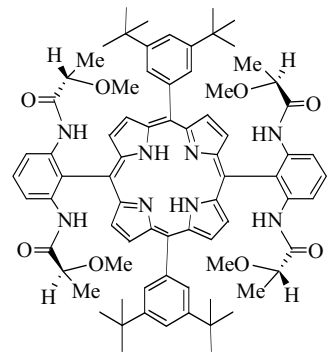

Porphyrin 3n (Table 1, entry 14). The general procedure was used to couple 5,15-bis(2,6dibromophenyl)-10,20-bis[3,5-di(tert-butyl)phenyl]porphyrin (0.058 g, $0.05 \mathrm{mmol})$ with (S)(-)-2-methoxypropionamide $(0.082 \mathrm{~g}, 0.8 \mathrm{mmol})$, using molecular sieves $(4 \mathrm{~A}, 0.1 \mathrm{~g})$, $\mathrm{Pd}(\mathrm{OAc})_{2}(0.004 \mathrm{~g}, 0.02 \mathrm{mmol})$, Xantphos $(0.023 \mathrm{~g}, 0.04 \mathrm{mmol})$, and $\mathrm{Cs}_{2} \mathrm{CO}_{3}(0.261 \mathrm{~g}, 0.8$ $\mathrm{mmol})$. The reaction was conducted in THF $(4 \mathrm{~mL})$ at $100{ }^{\circ} \mathrm{C}$ for $48 \mathrm{~h}$. The pure compound was isolated by flash column chromatography (silica gel, ethyl acetate:hexanes $(\mathrm{v} / \mathrm{v})=1: 2$ ) as purple solids $(0.045 \mathrm{~g}, 72 \%) .{ }^{1} \mathrm{H}$ NMR $\left(300 \mathrm{MHz}, \mathrm{CDCl}_{3}\right): \delta 8.90(\mathrm{~d}, J=4.8 \mathrm{~Hz}, 4 \mathrm{H})$, $8.79(\mathrm{~d}, J=4.8 \mathrm{~Hz}, 4 \mathrm{H}), 8.57(\mathrm{~d}, J=8.7 \mathrm{~Hz}, 4 \mathrm{H}), 7.94(\mathrm{~s}, 4 \mathrm{H}), 7.88(\mathrm{t}, J=8.7 \mathrm{~Hz}, 2 \mathrm{H}), 7.82$ $(\mathrm{s}, 6 \mathrm{H}), 3.08(\mathrm{q}, J=6.9 \mathrm{~Hz}, 4 \mathrm{H}), 1.52(\mathrm{~s}, 36 \mathrm{H}), 1.35(\mathrm{~s}, 12 \mathrm{H}), 0.65(\mathrm{~d}, J=7.2 \mathrm{~Hz}, 12 \mathrm{H})$, $-2.48(\mathrm{~s}, 2 \mathrm{H}) .{ }^{13} \mathrm{C}$ NMR $\left(75 \mathrm{MHz}, \mathrm{CDCl}_{3}\right): \delta 171.2,149.2,140.0,138.5,130.6,129.8$, 122.6, 122.3, 121.7, 117.0, 107.8, 78.1, 55.9, 35.0, 31.6, 17.8. UV-vis $\left(\mathrm{CH}_{2} \mathrm{Cl}_{2}\right), \lambda_{\max } \mathrm{nm}$ $(\log \varepsilon):$ 421(5.61), 516(4.31), 551(3.91), 592(3.80), 648(3.68). HRMS-MALDI ([M] $\left.]^{+}\right)$: calcd for $\mathrm{C}_{76} \mathrm{H}_{91} \mathrm{~N}_{8} \mathrm{O}_{8}$ 1243.6954, found 1243.6991 with an isotope distribution pattern that is the same as the calculated one. 


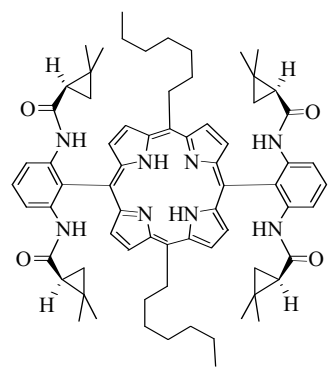

Porphyrin 30 (Table 1, entry 15). The general procedure was used to couple 5,15-bis(2,6dibromophenyl)-10,20-bisheptylporphyrin $(0.049 \mathrm{~g}, 0.05 \mathrm{mmol})$ with $(\mathrm{S})-(+)-2,2-$ dimethylcyclopropanecarboxamide $(0.184 \mathrm{~g}, 1.6 \mathrm{mmol})$, using $\mathrm{Pd}(\mathrm{OAc})_{2}(0.004 \mathrm{~g}, 0.02$ $\mathrm{mmol})$, Xantphos $(0.023 \mathrm{~g}, 0.04 \mathrm{mmol})$, and $\mathrm{Cs}_{2} \mathrm{CO}_{3}(0.261 \mathrm{~g}, 0.8 \mathrm{mmol})$. The reaction was conducted in THF $(4 \mathrm{~mL})$ at $100{ }^{\circ} \mathrm{C}$ for $60 \mathrm{~h}$. The pure compound was isolated by flash column chromatography (silica gel, ethyl acetate:hexanes $(\mathrm{v} / \mathrm{v})=1: 3)$ as purple solids $(0.043$ g, 74\%). ${ }^{1} \mathrm{H} \mathrm{NMR}\left(300 \mathrm{MHz}, \mathrm{CDCl}_{3}\right): \delta 9.56(\mathrm{~d}, J=4.8 \mathrm{~Hz}, 4 \mathrm{H}), 8.95(\mathrm{~d}, J=4.8 \mathrm{~Hz}, 4 \mathrm{H})$, 8.51 (broad, 4H), 7.87 (t, $J=8.1 \mathrm{~Hz}, 2 \mathrm{H}), 6.50$ (broad, 4H), $5.03(\mathrm{~m}, 4 \mathrm{H}), 2.55(\mathrm{~m}, 4 \mathrm{H}), 1.86$ $(\mathrm{m}, 4 \mathrm{H}), 1.37(\mathrm{~m}, 6 \mathrm{H}), 0.91(\mathrm{~s}, 12 \mathrm{H}), 0.70$ (broad, 4H), -0.04-0.19 (m, 20H), $-2.48(\mathrm{~s}, 2 \mathrm{H})$. ${ }^{13} \mathrm{C}$ NMR (75 MHz, $\left.\mathrm{CDCl}_{3}\right): \delta 169.7,139.3,131.2,130.3,130.0,121.4,117.4,107.5,39.0$, $35.2,31.8,30.5,29.3,28.9,26.3,22.7,22.3,20.4,18.2,14.1$. UV-vis $\left(\mathrm{CH}_{2} \mathrm{Cl}_{2}\right), \lambda_{\max } \mathrm{nm}(\log$ $\varepsilon)$ : 421(5.40), 517(4.16), 553(3.82), 594(3.62), 651(3.71). HRMS-MALDI ([M+H] $\left.]^{+}\right)$: calcd for $\mathrm{C}_{70} \mathrm{H}_{87} \mathrm{~N}_{8} \mathrm{O}_{4} 1103.6845$, found 1103.6871 with an isotope distribution pattern that is the same as the calculated one.

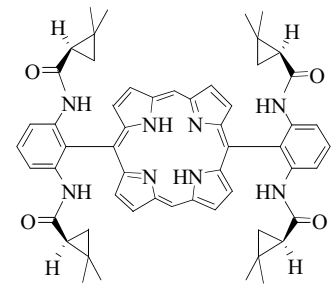

Porphyrin 3p (Table 1, entry 16). The general procedure was used to couple 5,15-bis(2,6dibromophenyl)porphyrin $(0.039 \mathrm{~g}, 0.05 \mathrm{mmol})$ with $(\mathrm{S})-(+)-2,2$-dimethylcyclopropane carboxamide $(0.181 \mathrm{~g}, 1.6 \mathrm{mmol})$, using $\mathrm{Pd}(\mathrm{OAc})_{2}(0.004 \mathrm{~g}, 0.02 \mathrm{mmol})$, Xantphos $(0.023 \mathrm{~g}$, $0.04 \mathrm{mmol})$, and $\mathrm{Cs}_{2} \mathrm{CO}_{3}(0.261 \mathrm{~g}, 0.8 \mathrm{mmol})$. The reaction was conducted in THF $(6 \mathrm{~mL})$ at $100{ }^{\circ} \mathrm{C}$ for $60 \mathrm{~h}$. The pure compound was isolated by flash column chromatography (silica gel, ethyl acetate:hexanes $(\mathrm{v} / \mathrm{v})=1: 1)$ as purple solids $(0.036 \mathrm{~g}, 79 \%) .{ }^{1} \mathrm{H}$ NMR $(300 \mathrm{MHz}$, $\left.\mathrm{CDCl}_{3}\right): \delta 10.44(\mathrm{~s}, 2 \mathrm{H}), 9.50(\mathrm{~d}, J=4.8 \mathrm{~Hz}, 4 \mathrm{H}), 9.08(\mathrm{~d}, J=4.8 \mathrm{~Hz}, 4 \mathrm{H}), 8.48$ (broad, 4H), $7.86(\mathrm{t}, J=8.7 \mathrm{~Hz}, 2 \mathrm{H}), 6.47$ (broad, 4H), 0.88 (s, 12H), 0.67 (broad, 4H), -0.14-0.13 (m, $20 \mathrm{H}),-3.05(\mathrm{~s}, 2 \mathrm{H}) .{ }^{13} \mathrm{C} \mathrm{NMR}\left(75 \mathrm{MHz}, \mathrm{CDCl}_{3}\right): \delta 169.7,147.2,146.4,139.3,133.7,130.7$, $130.5,117.7,108.0,106.3,28.9,26.2,22.4,20.4,18.2$. UV-vis $\left(\mathrm{CH}_{2} \mathrm{Cl}_{2}\right), \lambda_{\max } \mathrm{nm}(\log \varepsilon)$ : 409(5.31), 503(4.12), 536(3.74), 575(3.67), 628(3.40). HRMS-MALDI ([M+H] $\left.]^{+}\right)$: calcd for $\mathrm{C}_{56} \mathrm{H}_{59} \mathrm{~N}_{8} \mathrm{O}_{4} 907.4654$, found 907.4640 with an isotope distribution pattern that is the same as the calculated one.

General Procedures for Synthesis of Cobalt porphyrin complex. ${ }^{4}$ Free base porphyrin and anhydrous $\mathrm{CoCl}_{2}$ were placed in an oven-dried, resealable Schlenk tube. The tube was capped with a Teflon screwcap, evacuated, and backfilled with nitrogen. The screwcap was 
replaced with a rubber septum, 2,6-lutidine and dry THF were added via syringe. The tube was purged with nitrogen for 2 minutes, and then the septum was replaced with the Teflon screwcap. The tube was sealed, and its contents were heated with stirring. The resulting mixture was cooled to room temperature, taken up in ethyl acetate, and transferred to a separatory funnel. The mixture was washed with water 3 times and concentrated in vacuo.

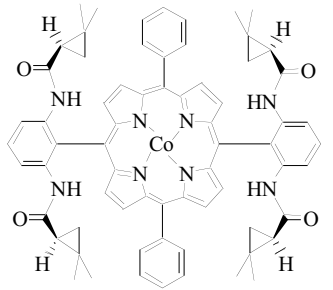

Cobalt porphyrin 4a (Table 1, entry 1). The general procedure was used for cobalt ion insertion. Free-base porphyrin $(0.066 \mathrm{~g})$, anhydrous $\mathrm{CoCl}_{2}(0.073 \mathrm{~g}), 2,6$-lutidine $(0.025 \mathrm{ml})$, and dry THF $(5 \mathrm{ml})$ were heated at $70^{\circ} \mathrm{C}$ under $\mathrm{N}_{2}$ for 16 hours. The resulting mixture was cooled to room temperature, taken up in ethyl acetate and transferred to a separatory funnel. The mixture was washed with water 3 times and concentrated in vacuo. The pure compound was obtained after flash column chromatography (silica gel, ethyl acetate:hexanes (v/v) = $1: 2)$ as a red solid $(0.061 \mathrm{~g}, 88 \%)$. UV-vis $\left(\mathrm{CH}_{2} \mathrm{Cl}_{2}\right), \lambda_{\max } \mathrm{nm}(\log \varepsilon)$ : 412(5.29), 529(4.06), 556(3.71). HRMS-EI $\left([\mathrm{M}]^{+}\right)$: calcd for $\mathrm{C}_{68} \mathrm{H}_{64} \mathrm{CoN}_{8} \mathrm{O}_{4}, 1115.4383$, found 1115.4376 with an isotope distribution pattern that is the same as the calculated one.

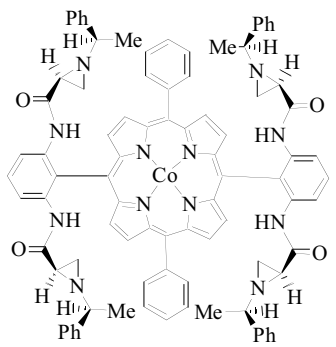

Cobalt porphyrin 4b (Table 1 , entry 2$)$. Free-base porphyrin $(0.019 \mathrm{~g})$, cobalt acetate tetrahydrate $(0.028 \mathrm{~g})$, and dry $\operatorname{DMF}(2 \mathrm{ml})$ were heated at $160^{\circ} \mathrm{C}$ under $\mathrm{N}_{2}$ for 3 hours. The resulting mixture was cooled to room temperature, taken up in ethyl acetate and transferred to a separatory funnel. The mixture was washed with water 3 times and concentrated in vacuo. The pure compound was obtained as a red solid $(0.017 \mathrm{~g}, 86 \%)$. UV-vis $\left(\mathrm{CH}_{2} \mathrm{Cl}_{2}\right)$, $\lambda_{\max } \mathrm{nm}(\log \varepsilon):$ 412(5.43), 528(4.30), 556(4.11), 615(3.71). HRMS-MALDI $\left([\mathrm{M}+\mathrm{H}]^{+}\right)$: calcd for $\mathrm{C}_{80} \mathrm{H}_{69} \mathrm{CoN}_{12} \mathrm{O}_{4} 1320.4891$, found 1320.3267 with an isotope distribution pattern that is the same as the calculated one.

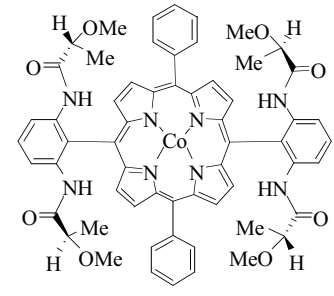


Cobalt porphyrin $4 \mathbf{c}$ (Table 1, entry 3 ). The general procedure was used for cobalt ion insertion. Free-base porphyrin $(0.025 \mathrm{~g})$, anhydrous $\mathrm{CoCl}_{2}(0.029 \mathrm{~g})$, 2,6-lutidine $(0.010 \mathrm{ml})$, and dry THF $(3 \mathrm{ml})$ were heated at $70^{\circ} \mathrm{C}$ under $\mathrm{N}_{2}$ for 15 hours. The resulting mixture was cooled to room temperature, taken up in ethyl acetate, and transferred to a separatory funnel. The mixture was washed with water 3 times and concentrated in vacuo. The pure compound was obtained as a red solid (0.025 g, 95\%). UV-vis $\left(\mathrm{CH}_{2} \mathrm{Cl}_{2}\right), \lambda_{\max } \mathrm{nm}(\log \varepsilon): 412(5.49)$, 528(4.25), 556(3.91). HRMS-MALDI ([M] $\left.]^{+}\right)$: calcd for $\mathrm{C}_{60} \mathrm{H}_{56} \mathrm{CoN}_{8} \mathrm{O}_{8} 1075.3548$, found 1075.3518 with an isotope distribution pattern that is the same as the calculated one.

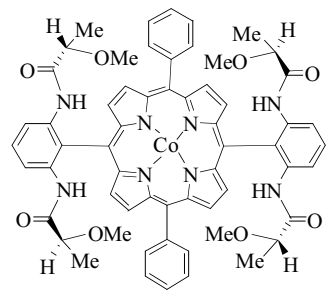

Cobalt porphyrin 4d (Table 1, entry 4). The general procedure was used for cobalt ion insertion. Free-base porphyrin $(0.020 \mathrm{~g})$, anhydrous $\mathrm{CoCl}_{2}(0.023 \mathrm{~g})$, 2,6-lutidine $(0.008 \mathrm{ml})$, and dry THF $(4 \mathrm{ml})$ were heated at $70^{\circ} \mathrm{C}$ under $\mathrm{N}_{2}$ for 14 hours. The resulting mixture was cooled to room temperature, taken up in ethyl acetate, and transferred to a separatory funnel. The mixture was washed with water 3 times and concentrated in vacuo. The pure compound was obtained as a red solid $(0.020 \mathrm{~g}, 95 \%)$. UV-vis $\left(\mathrm{CH}_{2} \mathrm{Cl}_{2}\right), \lambda_{\max } \mathrm{nm}(\log \varepsilon): 412(5.65)$, 528(4.39), 556(3.85). HRMS-MALDI ([M] $\left.]^{+}\right)$: calcd for $\mathrm{C}_{60} \mathrm{H}_{56} \mathrm{CoN}_{8} \mathrm{O}_{8} 1075.3548$, found 1075.3544 with an isotope distribution pattern that is the same as the calculated one.

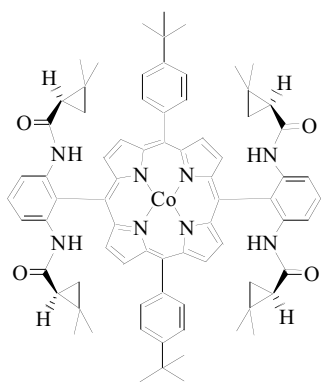

Cobalt porphyrin 4e (Table 1, entry 5 ). The general procedure was used for cobalt ion insertion. Free-base porphyrin $(0.045 \mathrm{~g})$, anhydrous $\mathrm{CoCl}_{2}(0.045 \mathrm{~g})$, 2,6-lutidine $(0.014 \mathrm{ml})$, and dry THF $(5 \mathrm{ml})$ were heated at $70^{\circ} \mathrm{C}$ under $\mathrm{N}_{2}$ for 12 hours. The resulting mixture was cooled to room temperature, taken up in ethyl acetate, and transferred to a separatory funnel. The mixture was washed with water 3 times and concentrated in vacuo. The pure compound was obtained after flash column chromatography (silica gel, ethyl acetate:hexanes $(\mathrm{v} / \mathrm{v})=$ $1: 4)$ as a red solid $(0.034 \mathrm{~g}, 72 \%)$. UV-vis $\left(\mathrm{CH}_{2} \mathrm{Cl}_{2}\right), \lambda_{\max } \mathrm{nm}(\log \varepsilon)$ : 413(5.48), 529(4.25), 554(3.92). HRMS-EI ([M] $]^{+}$): calcd for $\mathrm{C}_{76} \mathrm{H}_{80} \mathrm{CoN}_{8} \mathrm{O}_{4} 1227.5635$, found 1227.5593 with an isotope distribution pattern that is the same as the calculated one. 


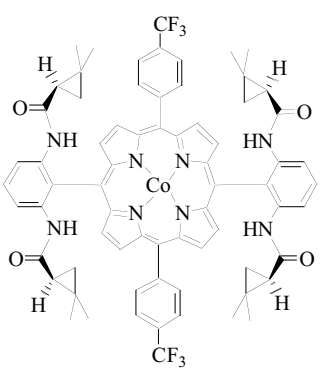

Cobalt porphyrin 4f (Table 1 , entry 6). The general procedure was used for cobalt ion insertion. Free-base porphyrin $(0.025 \mathrm{~g})$, anhydrous $\mathrm{CoCl}_{2}(0.022 \mathrm{~g}), 2,6$-lutidine $(0.008 \mathrm{ml})$, and dry THF $(2 \mathrm{ml})$ were heated at $70^{\circ} \mathrm{C}$ under $\mathrm{N}_{2}$ for 17 hours. The resulting mixture was cooled to room temperature, taken up in ethyl acetate, and transferred to a separatory funnel. The mixture was washed with water 3 times and concentrated in vacuo. The pure compound was obtained as a red solid $(0.025 \mathrm{~g}, 95 \%)$. UV-vis $\left(\mathrm{CH}_{2} \mathrm{Cl}_{2}\right), \lambda_{\max } \mathrm{nm}(\log \varepsilon): 420(4.85)$, 444(4.95), 523(4.11), 550(4.20). HRMS-EI ([M] $\left.]^{+}\right)$: calcd for $\mathrm{C}_{70} \mathrm{H}_{62} \mathrm{CoF}_{6} \mathrm{~N}_{8} \mathrm{O}_{4} 1251.4125$, found 1251.4085 with an isotope distribution pattern that is the same as the calculated one.

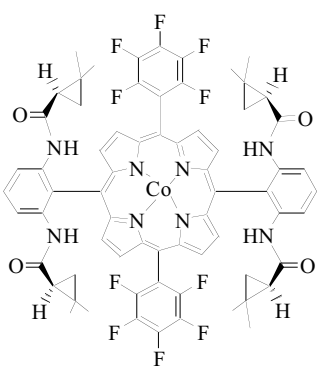

Cobalt porphyrin 4g (Table 1, entry 7). The general procedure was used for cobalt ion insertion. Free-base porphyrin $(0.010 \mathrm{~g})$, anhydrous $\mathrm{CoCl}_{2}(0.009 \mathrm{~g}), 2,6$-lutidine $(0.005 \mathrm{ml})$, and dry THF $(2 \mathrm{ml})$ were heated at $70^{\circ} \mathrm{C}$ under $\mathrm{N}_{2}$ for 14 hours. The resulting mixture was cooled to room temperature, taken up in ethyl acetate, and transferred to a separatory funnel. The mixture was washed with water 3 times and concentrated in vacuo. The pure compound was obtained as a red solid $(0.009 \mathrm{~g}, 86 \%)$. UV-vis $\left(\mathrm{CH}_{2} \mathrm{Cl}_{2}\right), \lambda_{\max } \mathrm{nm}(\log \varepsilon): 410(5.57)$, 443(4.70), 527(4.41), 556(4.23). HRMS-MALDI $\left([\mathrm{M}]^{+}\right)$: calcd for $\mathrm{C}_{68} \mathrm{H}_{54} \mathrm{CoF}_{10} \mathrm{~N}_{8} \mathrm{O}_{4}$ 1295.3435, found 1295.3459 with an isotope distribution pattern that is the same as the calculated one.

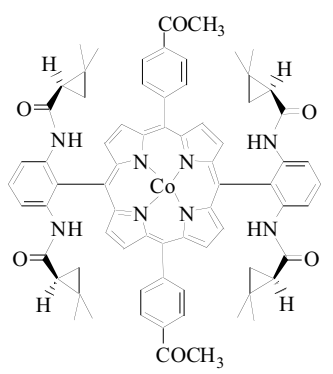

Cobalt porphyrin $4 \mathrm{~h}$ (Table 1 , entry 8 ). The general procedure was used for cobalt ion insertion. Free-base porphyrin $(0.030 \mathrm{~g})$, anhydrous $\mathrm{CoCl}_{2}(0.031 \mathrm{~g}), 2,6$-lutidine $(0.010 \mathrm{ml})$, and dry THF $(3 \mathrm{ml})$ were heated at $70^{\circ} \mathrm{C}$ under $\mathrm{N}_{2}$ for 12 hours. The resulting mixture was 
cooled to room temperature, taken up in ethyl acetate, and transferred to a separatory funnel. The mixture was washed with water 3 times and concentrated in vacuo. The pure compound was obtained after flash column chromatography (silica gel, ethyl acetate:hexanes $(\mathrm{v} / \mathrm{v})=$ $1: 1)$ as a red solid $(0.026 \mathrm{~g}, 83 \%)$. UV-vis $\left(\mathrm{CH}_{2} \mathrm{Cl}_{2}\right), \lambda_{\max } \mathrm{nm}(\log \varepsilon)$ : 413(5.53), 528(4.31), 553(3.98). HRMS-MALDI ([M] $]^{+}$): calcd for $\mathrm{C}_{72} \mathrm{H}_{68} \mathrm{CoN}_{8} \mathrm{O}_{6} 1199.4588$, found 1199.4572 with an isotope distribution pattern that is the same as the calculated one.

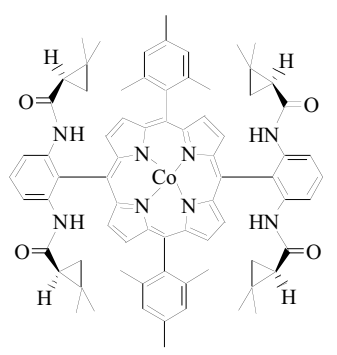

Cobalt porphyrin 4i (Table 1 , entry 9). The general procedure was used for cobalt ion insertion. Free-base porphyrin $(0.070 \mathrm{~g})$, anhydrous $\mathrm{CoCl}_{2}(0.071 \mathrm{~g})$, 2,6-lutidine $(0.024 \mathrm{ml})$, and dry THF $(5 \mathrm{ml})$ were heated at $70^{\circ} \mathrm{C}$ under $\mathrm{N}_{2}$ for 16 hours. The resulting mixture was cooled to room temperature, taken up in ethyl acetate, and transferred to a separatory funnel. The mixture was washed with water 3 times and concentrated in vacuo. The pure compound was obtained after flash column chromatography (silica gel, ethyl acetate:hexanes $(\mathrm{v} / \mathrm{v})=$ $1: 3)$ as a red solid $(0.067 \mathrm{~g}, 91 \%)$. UV-vis $\left(\mathrm{CH}_{2} \mathrm{Cl}_{2}\right), \lambda_{\max } \mathrm{nm}(\log \varepsilon)$ : 413(5.33), 528(4.09), 558(3.73). HRMS-EI $\left([\mathrm{M}]^{+}\right)$: calcd for $\mathrm{C}_{74} \mathrm{H}_{76} \mathrm{CoN}_{8} \mathrm{O}_{4}, 1199.5322$, found 1199.5320 with an isotope distribution pattern that is the same as the calculated one.

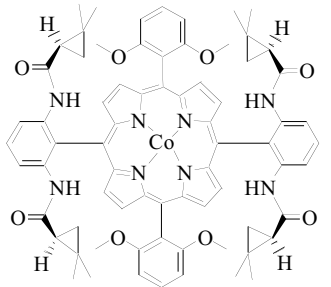

Cobalt porphyrin $4 \mathbf{j}$ (Table 1 , entry 10 ). The general procedure was used for cobalt ion insertion. Free-base porphyrin $(0.050 \mathrm{~g})$, anhydrous $\mathrm{CoCl}_{2}(0.044 \mathrm{~g})$, 2,6-lutidine $(0.015 \mathrm{ml})$, and dry THF $(3 \mathrm{ml})$ were heated at $70^{\circ} \mathrm{C}$ under $\mathrm{N}_{2}$ for 19 hours. The resulting mixture was cooled to room temperature, taken up in ethyl acetate, and transferred to a separatory funnel. The mixture was washed with water 3 times and concentrated in vacuo. The pure compound was obtained as a red solid $(0.050 \mathrm{~g}, 95 \%)$. UV-vis $\left(\mathrm{CH}_{2} \mathrm{Cl}_{2}\right), \lambda_{\max } \mathrm{nm}(\log \varepsilon): 413(5.18)$, 439(4.53), 532(4.09), 551(4.00). HRMS-EI ([M] $\left.]^{+}\right)$: calcd for $\mathrm{C}_{72} \mathrm{H}_{72} \mathrm{CoN}_{8} \mathrm{O}_{8}$ 1235.4805, found 1235.4794 with an isotope distribution pattern that is the same as the calculated one. 


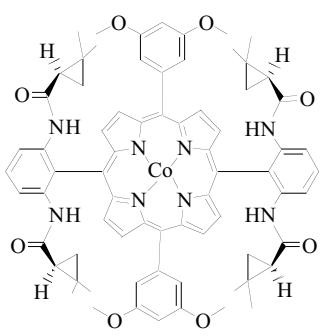

Cobalt porphyrin 4k (Table 1 , entry 11$)$. The general procedure was used for cobalt ion insertion. Free-base porphyrin (0.023 g), anhydrous $\mathrm{CoCl}_{2}(0.022 \mathrm{~g}), 2,6$-lutidine $(0.007 \mathrm{ml})$, and dry THF $(3 \mathrm{ml})$ were heated at $70^{\circ} \mathrm{C}$ under $\mathrm{N}_{2}$ for 15 hours. The resulting mixture was cooled to room temperature, taken up in ethyl acetate, and transferred to a separatory funnel. The mixture was washed with water 3 times and concentrated in vacuo. The pure compound was obtained as a red solid $(0.023 \mathrm{~g}, 96 \%)$. UV-vis $\left(\mathrm{CH}_{2} \mathrm{Cl}_{2}\right), \lambda_{\max } \mathrm{nm}(\log \varepsilon): 414(4.92)$, 445(4.66), 530(4.13), 553(4.11). HRMS-MALDI $\left([\mathrm{M}]^{+}\right)$: calcd for $\mathrm{C}_{72} \mathrm{H}_{72} \mathrm{CoN}_{8} \mathrm{O}_{8}$ 1235.4800, found 1235.4749 with an isotope distribution pattern that is the same as the calculated one.

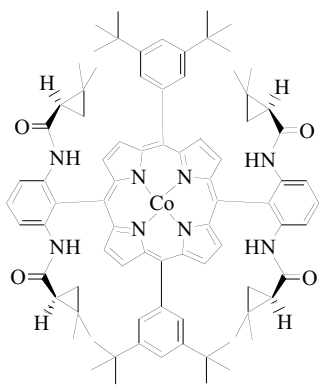

Cobalt porphyrin $4 \mathbf{l}$ (Table 1 , entry 12 ). The general procedure was used for cobalt ion insertion. Free-base porphyrin $(0.100 \mathrm{~g})$, anhydrous $\mathrm{CoCl}_{2}(0.080 \mathrm{~g}), 2,6$-lutidine $(0.027 \mathrm{ml})$, and dry THF $(5 \mathrm{ml})$ were heated at $70^{\circ} \mathrm{C}$ under $\mathrm{N}_{2}$ for 9 hours. The resulting mixture was cooled to room temperature, taken up in ethyl acetate, and transferred to a separatory funnel. The mixture was washed with water 3 times and concentrated in vacuo. The pure compound was obtained after flash column chromatography ( silica gel, ethyl acetate:hexanes $(\mathrm{v} / \mathrm{v})=$ $1: 4)$ as a red solid $(0.099 \mathrm{~g}, 91 \%)$. UV-vis $\left(\mathrm{CH}_{2} \mathrm{Cl}_{2}\right), \lambda_{\max } \mathrm{nm}(\log \varepsilon): 414(5.37), 529(4.14)$, 549(3.84). HRMS-EI ([M] $\left.]^{+}\right)$: calcd for $\mathrm{C}_{84} \mathrm{H}_{96} \mathrm{CoN}_{8} \mathrm{O}_{4} 1339.6887$, found 1339.6909 with an isotope distribution pattern that is the same as the calculated one.

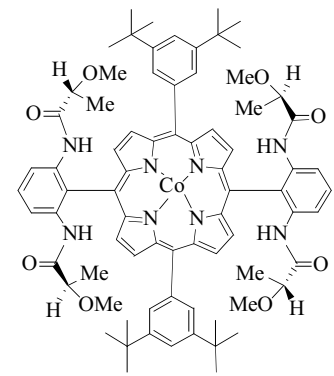

Cobalt porphyrin $4 \mathrm{~m}$ (Table 1, entry 13). The general procedure was used for cobalt ion insertion. Free-base porphyrin $(0.029 \mathrm{~g})$, anhydrous $\mathrm{CoCl}_{2}(0.026 \mathrm{~g}), 2,6$-lutidine $(0.010 \mathrm{ml})$, 
and dry THF $(3 \mathrm{ml})$ were heated at $70^{\circ} \mathrm{C}$ under $\mathrm{N}_{2}$ for 15 hours. The resulting mixture was cooled to room temperature, taken up in ethyl acetate, and transferred to a separatory funnel. The mixture was washed with water 3 times and concentrated in vacuo. The pure compound was obtained as a red solid $(0.029 \mathrm{~g}, 96 \%)$. UV-vis $\left(\mathrm{CH}_{2} \mathrm{Cl}_{2}\right), \lambda_{\max } \mathrm{nm}(\log \varepsilon)$ : 414(5.52), 529(4.23), 558(3.96). HRMS-MALDI ([M] $\left.]^{+}\right)$: calcd for $\mathrm{C}_{76} \mathrm{H}_{88} \mathrm{CoN}_{8} \mathrm{O}_{8} 1299.6052$, found 1299.6082 with an isotope distribution pattern that is the same as the calculated one.

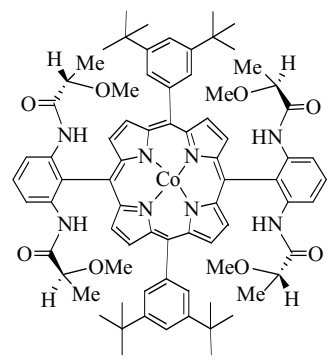

Cobalt porphyrin 4n (Table 1, entry 14). The general procedure was used for cobalt ion insertion. Free-base porphyrin $(0.030 \mathrm{~g})$, anhydrous $\mathrm{CoCl}_{2}(0.028 \mathrm{~g})$, 2,6-lutidine $(0.010 \mathrm{ml})$, and dry THF $(3 \mathrm{ml})$ were heated at $70^{\circ} \mathrm{C}$ under $\mathrm{N}_{2}$ for 15 hours. The resulting mixture was cooled to room temperature, taken up in ethyl acetate, and transferred to a separatory funnel. The mixture was washed with water 3 times and concentrated in vacuo. The pure compound was obtained as a red solid $(0.029 \mathrm{~g}, 92 \%)$. UV-vis $\left(\mathrm{CH}_{2} \mathrm{Cl}_{2}\right), \lambda_{\max } \mathrm{nm}(\log \varepsilon)$ : 414(5.54), 529(4.29), 557(3.94). HRMS-MALDI ([M] $\left.]^{+}\right)$: calcd for $\mathrm{C}_{76} \mathrm{H}_{88} \mathrm{CoN}_{8} \mathrm{O}_{8} 1299.6052$, found 1299.6070 with an isotope distribution pattern that is the same as the calculated one.

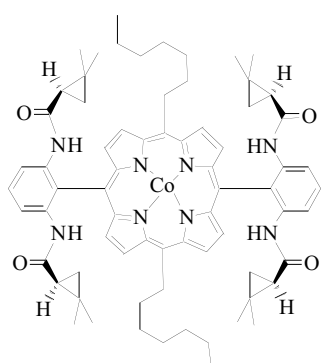

Cobalt porphyrin 4o (Table 1, entry 15). The general procedure was used for cobalt ion insertion. Free-base porphyrin $(0.030 \mathrm{~g})$, anhydrous $\mathrm{CoCl}_{2}(0.030 \mathrm{~g})$, 2,6-lutidine $(0.009 \mathrm{ml})$, and dry THF $(4 \mathrm{ml})$ were heated at $70^{\circ} \mathrm{C}$ under $\mathrm{N}_{2}$ for 16 hours. The resulting mixture was cooled to room temperature, taken up in ethyl acetate, and transferred to a separatory funnel. The mixture was washed with water 3 times and concentrated in vacuo. The pure compound was obtained as a red solid $(0.030 \mathrm{~g}, 95 \%)$. UV-vis $\left(\mathrm{CH}_{2} \mathrm{Cl}_{2}\right), \lambda_{\max } \mathrm{nm}(\log \varepsilon): 414(5.35)$, 443(4.37), 533(4.18), 560(3.89). HRMS-MALDI $\left([\mathrm{M}]^{+}\right)$: calcd for $\mathrm{C}_{70} \mathrm{H}_{84} \mathrm{CoN}_{8} \mathrm{O}_{4}$ 1159.5942 , found 1159.5927 with an isotope distribution pattern that is the same as the calculated one. 


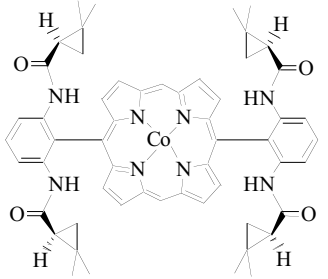

Cobalt porphyrin 4p (Table 1 , entry 16). The general procedure was used for cobalt ion insertion. Free-base porphyrin $(0.017 \mathrm{~g})$, anhydrous $\mathrm{CoCl}_{2}(0.022 \mathrm{~g}), 2,6$-lutidine $(0.007 \mathrm{ml})$, and dry THF $(3 \mathrm{ml})$ were heated at $70^{\circ} \mathrm{C}$ under $\mathrm{N}_{2}$ for 15 hours. The resulting mixture was cooled to room temperature, taken up in ethyl acetate, and transferred to a separatory funnel. The mixture was washed with water 3 times and concentrated in vacuo. The pure compound was obtained as a red solid $(0.017 \mathrm{~g}, 91 \%)$. UV-vis $\left(\mathrm{CH}_{2} \mathrm{Cl}_{2}\right), \lambda_{\max } \mathrm{nm}(\log \varepsilon): 404(5.27)$, 428(4.93), 461(4.62), 521(4.39), 547(4.41). HRMS-MALDI ([M] $\left.]^{+}\right)$: calcd for $\mathrm{C}_{56} \mathrm{H}_{56} \mathrm{CoN}_{8} \mathrm{O}_{4}$ 963.3751, found 963.3726 with an isotope distribution pattern that is the same as the calculated one.

General Procedures for Cyclopropanation of Styrene. Catalyst (1 mol \%) and DMAP were placed in an oven-dried, resealable Schlenk tube. The tube was capped with a Teflon screwcap, evacuated, and backfilled with nitrogen. The screwcap was replaced with a rubber septum, and 1.0 equivalent of styrene $(0.25 \mathrm{mmol})$ was added via syringe, followed by toluene $(0.5 \mathrm{~mL}), 1.2$ equivalents of diazo compound and toluene again $(0.5 \mathrm{~mL})$. The tube was purged with nitrogen for $1 \mathrm{~min}$ and its contents were stirred at room temperature. After the reaction finished, the resulting mixture was concentrated and the residue was purified by flash silica gel chromatography to give the product.

Ethyl 2-phenylcyclopropane-1-carboxylate ${ }^{5}{ }^{1} \mathrm{H}$ NMR $\left(300 \mathrm{MHz}, \mathrm{CDC1}_{3}\right)$ trans-isomer: $\delta$ 7.09-7.31 (m, 5H), 4.17 (q, $J=7.2 \mathrm{~Hz}, 2 \mathrm{H}), 2.52$ (ddd, $J=9.3,6.6,4.2 \mathrm{~Hz}, 1 \mathrm{H}), 1.90$ (ddd, $J$ $=8.7,5.4,4.5 \mathrm{~Hz}, 1 \mathrm{H}), 1.60(\mathrm{ddd}, J=9.0,5.1,4.2 \mathrm{~Hz}, 1 \mathrm{H}), 1.30(\mathrm{ddd}, J=8.4,6.6,4.8 \mathrm{~Hz}$, $1 \mathrm{H}), 1.28(\mathrm{t}, J=7.2 \mathrm{~Hz}, 3 \mathrm{H}) .{ }^{13} \mathrm{C} \mathrm{NMR}\left(75 \mathrm{MHz}, \mathrm{CDC1}_{3}\right)$ trans-isomer: $\delta$ 173.4, 140.1, 128.4, 126.4, 126.1, 60.7, 26.2, 24.2, 17.1, 14.3. ${ }^{1} \mathrm{H}$ NMR (300 MHz, $\left.\mathrm{CDC1}_{3}\right)$ cis-isomer: $\delta$ 7.18-7.28 (m, 5H), $3.88(\mathrm{q}, J=7.2 \mathrm{~Hz}, 2 \mathrm{H}), 2.59(\mathrm{~m}, 1 \mathrm{H}), 2.08(\mathrm{ddd}, J=9.0,7.8,5.6 \mathrm{~Hz}$, $1 \mathrm{H}), 1.72$ (ddd, $J=6.3,4.9,4.4 \mathrm{~Hz}, 1 \mathrm{H}), 1.32$ (ddd, $J=8.9,7.9,5.0 \mathrm{~Hz}, 1 \mathrm{H}), 0.97$ (t, $J=7.2$ $\mathrm{Hz}, 3 \mathrm{H}) .{ }^{13} \mathrm{C}$ NMR $\left(75 \mathrm{MHz}, \mathrm{CDC1}_{3}\right)$ cis-isomer: $\delta$ 170.9, 136.5, 129.2, 127.8, 126.6, 60.1, $25.4,21.7,14.0,11.1$.

tert-Butyl 2-phenylcyclopropane-1-carboxylate ${ }^{6}{ }^{1} \mathrm{H}$ NMR $\left(300 \mathrm{MHz}, \mathrm{CDC}_{3}\right)$ transisomer: $\delta$ 7.07-7.29 (m, 5H), $2.44(\mathrm{~m}, 1 \mathrm{H}), 1.82(\mathrm{~m}, 1 \mathrm{H}), 1.53(\mathrm{~m}, 1 \mathrm{H}), 1.46(\mathrm{~s}, 9 \mathrm{H}), 1.21(\mathrm{~m}$, 1H). $\left.{ }^{13} \mathrm{C} \mathrm{NMR} \mathrm{(75} \mathrm{MHz,} \mathrm{CDC1} 1_{3}\right)$ trans-isomer: $\delta$ 172.5, 140.5, 128.4, 126.3, 126.0, 80.5, 28.1, 26.0, 25.3, 17.0. ${ }^{1} \mathrm{H}$ NMR (300 MHz, $\left.\mathrm{CDC1}_{3}\right)$ cis-isomer: $\delta$ 7.17-7.27 (m, 5H), $2.52(\mathrm{~m}$, 1H), $1.99(\mathrm{~m}, 1 \mathrm{H}), 1.65(\mathrm{~m}, 1 \mathrm{H}), 1.24(\mathrm{~m}, 1 \mathrm{H}), 1.13(\mathrm{~s}, 9 \mathrm{H}) .{ }^{13} \mathrm{C}$ NMR $\left(75 \mathrm{MHz}, \mathrm{CDC} 1_{3}\right) \mathrm{cis}^{-}$ isomer: $\delta 170.1,136.8,129.5,127.8,126.5,80.0,27.7,25.0,22.7,10.5$. 


\section{Reference}

1) Lindsey, J. S.; Wagner, R. W. J. Org. Chem. 1989, 54, 828.

2) Lindsey, J. S. In The Porphyrin Handbook; Kadish, K. M., Smith, K. M., Guilard, R., Eds.; Academic Press: San Diego, CA, 2000; Vol. 1; pp 45-118.

3) Gao, G.-Y.; Chen, Y.; Zhang, X. P. Org. Lett. 2004, 6, 1837.

4) (a) Tsuchida, E.; Hasegawa, E.; Komatsu, T.; Nakata, T.; Nishide, H. Chem. Lett. 1990, (3), 389. (b) Tsuchida, E.; Komatsu, T.; Hasegawa, E.; Nishide, H. J. Chem. Soc.-Dalton Trans. 1990, 2713. (c) Komatsu, T.; Hasegawa, E.; Nishide, H.; Tsuchida, E. J. Chem. Soc.-Chem. Commun. 1990, 66.

5) Sanders, C. J.; Gillrspie, K. M.; Scott, P. Tetrahedron Asymmetry 2001, 12, 1055.

6) Niimi, T.; Uchida, T.; Irie, R.; Katsuki, T. Adv. Synth. Catal. 2001, 343, 79. 